\title{
Studies of the modal properties of Circularly Photonic Crystal Fiber (C-PCF) for high power applications
}

\author{
Partha Sona Maji ${ }^{1 *}$ and Partha Roy Chaudhuri ${ }^{1}$ \\ Department of Physics, Indian Institute of Technology, Kharagpur, India 721302. \\ *parthamaji@phy.iitkgp.ernet.in
}

\begin{abstract}
:
The guiding properties of a new type of photonic crystal fibers where air-holes are arranged in a circular pattern (C-PCF) with a silica matrix have been investigated. The dispersion properties of the fiber with different spacing of circle and air-hole diameter have been studied in detail. It is shown that C-PCFs with smaller values of radius and higher air-filling fraction can be used as dispersion compensating fiber. A comparison between fibers with circular and triangular lattice has also been performed, taking into account the dispersion properties and the effective area in the wavelength range between $1200 \mathrm{~nm}$ and $1600 \mathrm{~nm}$. C-PCF can better compensate the inline dispersion for both single wavelength and broadband wavelength applications which is a unique property not observed by regular triangular-lattice or squarelattice PCFs. The fiber provides higher effective area, making it a better candidate for high power accumulations in the core of the fiber. The fiber also shows red-shifting of the first zero dispersion wavelength (ZDW), flatter dispersion slope and lower Group Velocity Dispersion (GVD) in the normal dispersion region thereby making it a better candidate for high power nonlinear applications like supercontinuum generation, soliton pulse propagation etc. With the above advantages, we have considered a series study of these circular-lattice structures for various geometrical parameters and temporal pulses in order to explore the characteristics of broadband supercontinuum generation. This design study for high power supercontinuum generation will be very helpful for potential application of new sources in various fields like astronomy, climatology, spectroscopy optical tomography and sensing etc. to name a few.
\end{abstract}

Keywords: Photonic Crystal Fibers; Microstructured fibers; Dispersion compensation devices; Supercontinuum generation.

\section{Introduction:}

Photonic Crystal Fibers (PCFs) where air-holes are arranged in either triangular lattice of square lattice with a silica background have been extensively studied in details for different applications. They posses some novel guiding properties, related to the geometric characteristics of the air-holes in their crosssection, and have been successfully exploited in different application [1-2]. Most of the air-holes in the PCFs cladding have been arranged either in a periodic triangular or periodic square orientation. The modal properties, in particular, the dispersion properties of the above types of PCFs can be altered by varying the hole-to-hole spacing $(\Lambda)$ and the air-hole diameter $(d)$ with air-hole fraction being $d / \Lambda[3,4]$. Both types of PCFs with a silica background can be successfully implemented to compensate the positive dispersion parameter and dispersion slope of the existing inline fibers [3, 4]. These fibers can be engineered for designing ultra-flattened near zero dispersion [5-12] or can be engineered to have ultra negative dispersion values near the communication wavelength [13-19].

Photonic Crystal with air-holes arranged in circular layer arrangements have been investigated for different applications [20-27] such as studying thermal properties for electrical driving [20], high

(c) 2016. This manuscript version is made available under the Elsevier user license http://www.elsevier.com/open-access/userlicense/1.0/ 
transmission waveguides [21], high quality factor microcavity lasers with isotropic photonic band gap effect [22], localization of electromagnetic waves [23] and achieving ultra-flat dispersion [24] etc. Argyros et al [28] realized PCF with circular air-holes arrangement with polymer background. Though the above results demonstrate few works have been accomplished, still a detail guiding and modal properties of CPCF with silica background has not yet been explored for its potential in the communication wavelength window or Infrared (IR) or far-IR applications.

In this work, different guiding and modal properties, especially the dispersion properties of PCFs with airhole arranged in a circular pattern have been investigated. It is interesting to analyze how a regular circular air-hole disposition, different from the triangular one, usually studied so far, can affect the characteristics of the guided mode. Moreover, it is important to understand in which terms all the results previously obtained for the triangular PCFs can be applied to the circular-lattice ones. Our numerical investigations reveal that C-PCF can better compensate the inline dispersion for both single wavelength and broadband applications. C-PCF has also been found out to be a better candidate for high power applications like wide band supercontinuum generation (SCG) because of its unique properties like (i) larger mode area that can accumulate high power in the core, (ii) red-shifting of zero dispersion wavelength $(Z D W)$ that is preferable towards mid-IR SCG specially with chalcogenide materials, (iii) lower dispersion slope in the anomalous dispersion region that leads to a broader spectrum and (iv) low Group velocity Dispersion GVD in the normal dispersion region that leads to higher Dispersion Length $\left(L_{D}\right)$ and higher degree of solitonic interaction. With the above advantages for a desired application with a target broadband and spectral power, we have explored the SCG property of the designed fiber by varying different geometrical parameters, considering the influence of varying pulse widths, peak powers and pumping wavelengths in detail.

\section{Geometry of the studied structure and modal analysis:}

The schematic diagram of the C-PCF is shown in Fig. 1 with the air-hole diameter $d$ and $R$ is the spacing between two neighboring air-holes in a particular circle. Also, $\mathrm{n} R$ is the distance from the center of the fiber to each air-hole of $\mathrm{n}^{\text {th }}$ circle. Subsequently, the distance from the center to the first circle to the center is $R$ whereas; the distance from the center to the $2^{\text {nd }}$ circle is $2 R$ and so on. The C-PCF with circular symmetry is constructed by repeating the circular unit around the core center. The circle has $6 n$ (with $n=1,2,3$ etc for first, second and third ring of air-holes) no of air-holes in a particular air-hole ring. The angular spacing between any two consecutive air-holes in a circle can be given by $\theta=\frac{360}{6 n}$, where $n=1,2,3$ etc for first, second, third layer of the circles forming the cladding cross-section. The structure has been designed based on $C_{2 v}$ symmetry. We compare the propagation characteristics of CPCF with triangular-lattice PCF for the structural parameters, the pitch ' $\Lambda$ ' (hole to hole distance of regular triangular-lattice PCF) was found to have the direct equivalence with ' $R$ ' (the radius of the circle and the distance between two consecutive circles). Thus, employing the above analogy under the same air-hole diameter $d, d / \Lambda$ (the air-filling fraction) for triangular-PCF can be compared with $d / R$ of C-PCF. Another important geometrical parameter is air-filling fraction $f$. The corresponding value for triangularlattice PCF is $f_{\Delta}=\left(\pi d^{2}\right) /\left(2 \sqrt{3} \Lambda^{2}\right)=0.9069\left(d^{2} / \Lambda^{2}\right)$, where as for the CPCF the value 
is $f_{\square} \approx\left(6 \pi d^{2}\right) /\left(8 R^{2}\right) \approx 0.75\left(d^{2} / R^{2}\right)$. So C-PCF is having air-filling factor $82 \%$ to that of triangular lattice PCF. In this type of PCF structure, the propagation modes can exist due to the modified Total Internal Reflection (TIR) mechanism, i.e., the lower index of the air-filled cladding and higher index of the silica fiber core. The modal fields are calculated using CUDOS MOF Utilities [29] that simulates PCFs using the multipole method [30-31]. Here we would like to mention that we are modeling a PCF structure with un-doped silica glass having holes filled with vacuum. The numerical calculations related to dispersion parameter $(D)$ and supercontinuum spectra are calculated with MATLAB ${ }^{\circledR}$. The guiding properties of the C-PCF have been studied in the following section by taking into consideration with various combinations of radius of the circle and air-hole diameter. We discussed the modal properties in the subsequent sections as follows.

\section{3: Dispersion properties}

PCFs possess the attractive property of great controllability in waveguide dispersion [3, 4]. Proper management of waveguide dispersion in PCFs is an important issue for applications to optical communications, dispersion compensation, and non-linear applications etc. The dispersion property for PCFs with regular triangular-lattice and square-lattice can be controlled by varying the $d$ and $\Lambda[3,4]$. Similarly, for C-PCF the dispersion can be controlled by varying the radius of the circle $R$ and air-hole diameter $d$. We have calculated the dispersion parameter $(D)$ of the structures through Eqn. (1).

$$
D=-\frac{\lambda}{c} \frac{d^{2} \operatorname{Re}\left[n_{e f f}\right]}{d \lambda^{2}}
$$

Here $\operatorname{Re}\left[\mathrm{n}_{\text {eff }}\right.$ is the real part of the effective indices obtained through simulations and $c$ is the velocity of light in vacuum. The confinement loss or the propagation loss for the structures has been calculated through Eqn. (2).

$$
L=\frac{2 \pi}{\lambda} \frac{20}{\ln (10)} 10^{6} \operatorname{Im}\left(n_{e f f}\right) d B / m
$$

Where $\operatorname{Im}\left(n_{\text {eff }}\right)$ is the imaginary part of the effective indices (obtained from the simulations) and $\lambda$ in micrometer.

The chromatic dispersion of the background material silica has been taken into account through Sellmeier's equation. The influence of the geometric parameters $R$ and $d$ has been investigated considering the C-PCFs with three layers of air holes in the cladding. In our study, we have considered $d / R$ varying from 0.3 to 0.9 in the steps of 0.1 . Figure 2 shows the variation of the $D$ parameter for $R$ values of $1 \mu \mathrm{m}, 2 \mu \mathrm{m}$ and $3 \mu \mathrm{m}$. From the figures it is clear that for the lowest values of $\mathrm{R}(\mathrm{i} . \mathrm{e} .1 \mu \mathrm{m})$ all the PCFs have negative value of dispersion parameter in the $C$ band i.e. around $1550 \mathrm{~nm}$. This is because of the fact that for smaller value of core diameter, the waveguide dispersion dominates the material one [3, 4]. It is also clear from Fig. 2(a) that the magnitude of the dispersion parameter increases with the increase of air-hole diameter. The figure clearly reveals that for smaller values of $d / R(\leq 0.5)$ the dispersion slope is always positive making it not suitable for dispersion compensation. For $d / R=0.6$, the dispersion slope is initially negative and then it becomes positive for higher wavelength range. For $d / R \geq$ 0.7, the dispersion slope is always negative through the wavelength range considered especially around wavelength of $1550 \mathrm{~nm}$. So C-PCF with $R=1 \mu \mathrm{m}$ and higher $d / R$ values can be useful for dispersion compensation. When $\Lambda$ becomes larger, the effect of waveguide dispersion decreases and material 
dispersion dominates the dispersion for both regular triangular lattice PCFs [3] and for square lattice PCF [4]. The same is confirmed for the CPCFs in Fig. 2(b) and Fig. 2(c) as we increase $R$ values to $2 \mu \mathrm{m}$ and 3 $\mu \mathrm{m}$ respectively. The dispersion parameters for all these PCFs become positive, independent of the $d / R$. It is also interesting to notice that as we increase the value of $R$, the dispersion slope of the curves becomes more and more positive. Moreover, a change of $d / R$ values causes only a small difference for higher values of dispersion parameter for higher values of $R=3 \mu \mathrm{m}$. It is also interesting to notice that for almost all values of $d / R$ the dispersion curve is quite flat for the whole wavelength range considered for $R=2 \mu \mathrm{m}$.

The variation of $D$ parameter for a change in radius of circle $(R)$ for a fixed $d / R$ has been discussed in Fig. 3(a) where we have considered $d / R=0.7$ (although the pattern are the same for all possible values of $d / R$ $\geq 0.7$ we have studied) and varying $R$ from $1 \mu \mathrm{m}$ to $3 \mu \mathrm{m}$ in a step of $0.5 \mu \mathrm{m}$. Most remarkable feature of the figure is when we change $R$ from $1 \mu \mathrm{m}$ to $1.5 \mu \mathrm{m}$, the dispersion parameter changes drastically. To better map the dispersion in this region of rapid transition for a change of $R$ from $1 \mu \mathrm{m}$ to $1.5 \mu \mathrm{m}$, we have plotted an extended study where the $R$ values are changed by $0.1 \mu \mathrm{m}$ as shown in Fig. 3(b). The graph clearly presents the steady variation of the dispersion parameter which seems to be rapid from Fig. 3(a). The dispersion slope is negative for $R=1 \mu \mathrm{m}$ to $1.5 \mu \mathrm{m}$, whereas the dispersion slope becomes positive for all the other cases. For all values of $R \geq 1.5 \mu \mathrm{m}$ the slope of the dispersion curve is always positive.

\section{4: Comparison between the C-PCF with triangular-lattice PCF}

\section{(a)Dispersion properties}

A comparison between the triangular-lattice PCFs and C-PCFs for guiding and dispersion properties has been performed. For this purpose, three air-hole rings for both types of fibers with $\Lambda=1 \mu \mathrm{m}$ and $d / \Lambda=$ 0.9 and equivalent $R=1 \mu \mathrm{m}$ and $d / R=0.9$ for C-PCF geometry were considered. Both the fibers can be used for dispersion compensating with negative dispersion around the communication wavelength as shown in Fig. 4(a). As can be seen from the figure, the C-PCFs have higher value of negative dispersion in comparison to the triangular-lattice PCFs. So, we need shorter length of C-PCF than the triangular one to completely compensate the dispersion at any wavelength, especially of the nonzero dispersion fiber (NZDF) at $1550 \mathrm{~nm}$. So C-PCF can compensate the inline dispersion some 10 times more than that the dispersion compensating fibers $(D C F s)$. The most interesting fact is that the C-PCFs can better compensate the positive dispersion of a NZDF in a wide wavelength range because of its lower value of dispersion slope around $1550 \mathrm{~nm}$. The relative dispersion slope $(R D S)$ of the C-PCF one at $1550 \mathrm{~nm}$ is $0.00357 \mathrm{~nm}^{-1}$ where as for the triangular one the value is $0.00461 \mathrm{~nm}^{-1}$. Taking into consideration that existing inline fibers from corning SMF-28 is having $R D S$ value $0.0036 \mathrm{~nm}^{-1}$ at $1550 \mathrm{~nm}$, CPCF with $R=$ $1.0 \mu \mathrm{m}$ with $d / R=0.9$ can better compensate the online dispersion both for a single wavelength as well as broadband dispersion that accumulates due to the broadening of pulse for a long haul communication. A better analysis for broadband dispersion compensation can be performed with the Compensation Ratio (CR) which is defined through Eqn. (3) [32].

$$
C R(\lambda)=\frac{D_{S M F}(\lambda)}{D_{S M F}} \frac{D_{D C F}}{D_{D C F}(\lambda)}
$$

with the value of $C R$ at $1550 \mathrm{~nm}$ to be unity because $L_{S M F}$ and $L_{D C F}$ are chosen such that they perfectly 
compensate the dispersion at $1550 \mathrm{~nm}$ according to Eqn. (4).

$$
D_{S M F} L_{S M F}+D_{D C F} L_{D C F}=0
$$

The value of $D_{S M F}$ is calculated through Eqn. (5) [33] with a zero dispersion wavelength $\lambda_{0}$ of $1311.5 \mathrm{~nm}$ and a zero dispersion slope $S_{0}$ of $0.092 \mathrm{ps} \mathrm{nm}^{-2} \mathrm{~km}^{-1}$

$$
D(\lambda)=\frac{S_{0}}{4}\left[\lambda-\frac{\lambda_{0}^{4}}{\lambda^{3}}\right]
$$

The calculated CR for the C-PCF with $R=1 \mu \mathrm{m}$ and $d / R=0.9$ at $1500 \mathrm{~nm}$ and $1600 \mathrm{~nm}$ are found to be of 1.005 and 0.988 which are very much closer to the optimum value of 1 whereas the value with triangular PCF comes out to be 1.066 and 0.941 respectively. So, C-PCF can better compensate the dispersion for a broadband wavelength range specially covering the $S, C$ and $L$ window of communication wavelength. This property is unique as neither the regular triangular-lattice nor square-lattice PCF posses the advantages of single wavelength and broadband dispersion compensation simultaneously together. The comparison of the dispersion properties between the C-PCF and T-PCF has been performed in another different way where we choose a T-PCF with a different adjustable parameter which gives same dispersion at $1550 \mathrm{~nm}$ with the above C-PCF with $R=1 \mu \mathrm{m}$ and $d / R=0.9$. Figure 4(b) shows the typical comparison between the two structures that provide same dispersion at $1550 \mathrm{~nm}$ with a T-PCF with $\Lambda=$ $0.905 \mu \mathrm{m}$ and $d / \Lambda=0.9$. Although the two structures provide same dispersion around $1550 \mathrm{~nm}$, the corresponding RDS of the new TPCF was found out to be $0.00287 \mathrm{~nm}^{-1}$, whereas for C-PCF the corresponding value was $0.00357 \mathrm{~nm}^{-1}$. The CR of this T-PCF was found out to be 0.965 at $1500 \mathrm{~nm}$ and 1.023 at $1600 \mathrm{~nm}$ respectively. So based upon the above findings of RDS and CR value of the new TPCF having the same dispersion around $1550 \mathrm{~nm}$, we can obviously find out the superiority of our design for both single wavelength and broadband dispersion compensation.

\section{(b) Effective area}

The effective area for the C-PCFs are still small, being lower than $2.5 \mu \mathrm{m}^{2}$ in the entire whole wavelength considered, as can be seen from Fig. 5(a) with $R=1.0 \mu \mathrm{m}$ with $d / R=0.9$. But this value is still higher than the triangular lattice one. For example, the effective area for the circular-lattice with same $d$ and $R$ has a value approximately $10 \%$ greater than the triangular one at the wavelength of $1550 \mathrm{~nm}$. The difference can be explained from the air-filling fraction $f_{\square} \approx\left(6 \pi d^{2}\right) /\left(8 R^{2}\right) \approx 0.75\left(d^{2} / R^{2}\right)$ for the CPCF which

is almost $82 \%$ than that of the triangular one whose $f_{\Delta}=\left(\pi d^{2}\right) /\left(2 \sqrt{3} \Lambda^{2}\right)=0.9069\left(d^{2} / \Lambda^{2}\right)$. As a consequence of the filling fraction the C-PCFs provide higher values of average refractive index of the cladding that means a lower step index, which subsequently results in a lower field confinement. Higher values of effective area for specific applications can be obtained with larger $R$ and smaller $d / R$ as shown in Fig. 9. We have also plotted the Effective area variation corresponding to Fig. 4(b) which is for same dispersion around 1550nm as shown in Fig 5(b). The figure presents a different nature where for smaller wavelengths, the effective area for the C-PCF is smaller however for higher wavelengths (including at $1550 \mathrm{~nm}$ ) the effective area of the same structure is found out to be higher. In a different comparison of 
the $\mathrm{A}_{\text {eff }}$ variation of the two designs, we have optimized a different T-PCF with $\Lambda=1.05 \mu \mathrm{m}$ and $d / \Lambda=$ 0.9 which demonstrate equal values of effective area around $1550 \mathrm{~nm}$ with the previous C-PCF (with $R=$ $1.0 \mu \mathrm{m}$ with $d / R=0.9$ ) as shown in Fig. 6(a). For higher wavelengths, the C-PCF geometry is having higher $\mathrm{A}_{\text {eff }}$ whereas the reverse can be found out for smaller wavelengths. It has been observed that the slope of the $\mathrm{A}_{\text {eff }}$ curve for C-PCF is always higher than the T-PCF for all the studied cases. The corresponding dispersion nature of the TPCF along with the CPCF has been presented in Fig. 6(b) with a dispersion value of $-189.41 \mathrm{ps} / \mathrm{nm} / \mathrm{km}$ around $1550 \mathrm{~nm}$ of wavelength with the corresponding RDS of $0.00612 \mathrm{~nm}^{-1}$ for the T-PCF. The corresponding value of CR for this structure is found out to be 1.174 at $1500 \mathrm{~nm}$ and 0.884 at $1600 \mathrm{~nm}$ respectively. So, even with a matched $\mathrm{A}_{\text {eff }}$, the dispersion nature turns out to be better for C-PCF than the T-PCF for both single wavelength and broadband applications.

\section{(c) Propagation loss of the structure}

Propagation loss of the circular lattice PCF in comparison with the triangular lattice PCF is presented in this section. The propagation loss of the PCF geometry is calculated with Eqn. (2). Figure 7(a) presents a comparison study of the propagation loss of C-PCF with $R=1 \mu \mathrm{m}$ and $d / R=0.9$ and triangular lattice with $\Lambda=1 \mu \mathrm{m}$ and $d / \Lambda=0.9$. It can be observed that for similar value of fiber parameters, the loss suffered by C-PCF is more than that of the equivalent triangular-lattice one. This can be understood based on the concept of the air-filling fraction. C-PCF with smaller air-filling fraction provide higher values of average refractive index of the cladding giving rise to a lower step index, which subsequently results in a lower field confinement and higher propagation loss [3-4].

Propagation loss of C-PCP with $R=1 \mu \mathrm{m}$ and $d / R=0.9$ has also been compared with the adjusted triangular-lattice PCF which demonstrate same dispersion $(D)$ around $1550 \mathrm{~nm}$ (Fig. 4(b) with $\Lambda=$

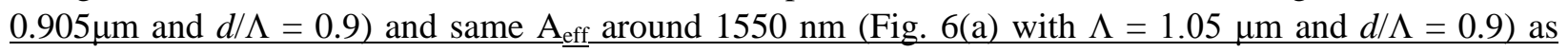
shown in Fig. 7 (b). It can be observed that the loss with smaller $\Lambda$ (with same dispersion around $1550 \mathrm{~nm}$ Fig. 4(b)) is higher and the loss with higher $\Lambda$ (with equal effective area around $1550 \mathrm{~nm}$ ) is lower than the CPCF with $R=1 \mu \mathrm{m}$ and $d / R=0.9$. The fact is quite obvious since increasing $\Lambda$ keeping same airfilling fraction, increases the size of the core which implies dominant material dispersion and lesser waveguide dispersion and consequently results in higher step index, which subsequently results in a higher field confinement and lower propagation loss [3-4].

\section{(c) Modal properties in single mode region}

A final analysis on the properties of both types of PCFs is reported in Fig. 8 and Fig. 9 for different values of $\Lambda$ ( $R$ for C-PCF) namely $2 \mu \mathrm{m}$ and $3 \mu \mathrm{m}$. We have considered $d / \Lambda=0.4$ for our study such that the triangular-PCFs remain single-mode in the whole wavelength considered even with higher values of $\Lambda$. An interesting fact can be observed from the figures that C-PCF has negative value of dispersion with negative slope at the $\mathrm{C}$-band of wavelength whereas for triangular-PCF the dispersion value is positive for lower values of $\Lambda$ (or $R$ for C-PCF) (i.e. $2 \mu \mathrm{m}$ ). For higher values of $\Lambda$ (or $R$ for C-PCF) (i.e. $3 \mu \mathrm{m}$ ) both the PCFs have positive dispersion slope with C-PCF having lower $D$ values than triangular PCF. The dispersion slope has been affected slightly with the change of geometrical characteristics of two different types of lattices. With the increase of the $\Lambda$ (or $R$ for CPCF) to $3 \mu \mathrm{m}$, the slope is always positive for both the cases but the slope is flatter for C-PCF than triangular PCF. PCFs with circular arrangement of airholes always have higher effective area than triangular one for both the value of $\Lambda$ (or equivalent $R$ values for C-PCF), making it a better option for high power accumulation in the core of the PCF needed for high 
power applications like supercontinuum generation, four wave mixing, parametric amplification and soliton pulse propagation etc. Higher Effective area will also be attractive for guiding and delivering high laser power because of enhanced threshold power limit for material damages to occur.

Next, we have considered $\Lambda$ ( $R$ for C-PCF $)=3 \mu \mathrm{m}$ with $d / \Lambda($ or $d / R)=0.3$ such that the structures remain single-mode for smaller wavelength (say, from $\lambda=0.8 \mu \mathrm{m}$ ). The dispersion parameter for the above cases for both types of PCFs is presented in Fig. 10. The results provide some important findings related to high power applications. With similar structural parameters, the value of first ZDW (shown by two dotted lined in the figure) for C-PCF has been red-shifted which is preferable towards mid-IR SC generation specially using non-silica high-nonlinear PCFs. Significantly, the dispersion profile of this PCF shows a lesser slope (i.e. the curve is less flat) than the normal dispersion characteristics revealed by similar type regular triangular-lattice PCFs. The lower slope in dispersion curve leads to broadening of the generated broadband supercontinuum pulse. Furthermore, its low Group velocity Dispersion (GVD) at anomalous dispersion region corresponds to higher Dispersion Length $\left(L_{D}\right)$ and higher degree of solitonic interaction. With the above advantages for a desired application with a target broadband and spectral power, we have explored the (SCG) property of the designed fiber by varying different geometrical parameters, considering the influence of varying pulse widths, peak powers and pumping wavelengths as discussed in the next section.

\section{Supercontinuum generation (SCG) with silica based C-PCF}

Designing SCG requires information related to the nonlinearity and other related issues like dispersion profile of the fiber [34-35]. Nonlinear coefficient is one of the most important parameters for SCG and has been calculated using the following equation,

$$
\gamma=\frac{2 \pi n_{2}}{\lambda A_{e f f}}
$$

where $n_{2}$ is the non-linear refractive indices of the material (silica here) and $A_{\text {eff }}$ is the effective area at that operating wavelength. The above data of a PCF so obtained are then used as input to the envelope based nonlinear Schrödinger equation (NLSE) which describes the pulse propagation through the PCF taking into account the contributions from the nonlinear effects, namely, self-phase modulation, Raman scattering, four-wave mixing. Eq. (7) represents the NLSE [35] for slowly varying pulse envelope $A(z, T)$ in the retarded time frame $T$

$$
\begin{aligned}
& \frac{\partial A}{\partial Z}+\frac{\alpha}{2} A-\sum_{n \geq 2} \frac{i^{n+1}}{n !} \beta n \frac{\partial^{n} A}{\partial T^{n}}= \\
& i \gamma\left(1-f_{R}\right)\left([A]^{2} A-\frac{i}{w_{0}} \frac{\partial}{\partial T}\left([A]^{2} A\right)+i \gamma f_{R}\left(1+\frac{i}{w_{0}}\right)\left(A \int_{0}^{\infty} h_{R}(\tau)|A(z, T-\tau)|^{2} \partial \tau\right)\right.
\end{aligned}
$$

where $\alpha$ is the loss coefficient, $\beta_{n}$, the $\mathrm{n}^{\text {th }}$ order dispersion, $\omega_{0}$, the input pulse frequency, $\tau$, the present time frame and $f_{R}$ is the fractional contribution due to delayed Raman function $h_{R}(\tau)$ [36]. NLSE is solved numerically by using the split-step Fourier transform based beam propagation code developed by COSTP11 [37]. In all the following cases, we have considered the pulse to be $\operatorname{sech}^{2}$ type with different values of Full Width at Half Maximum (FWHM). 
We present a detailed analysis of SC generation with C-PCF with different fused silica glass host showing the capability of such PCF as a SC source in visible to mid infrared region with femto-second (fs) laser pulse. We have considered a series study of these circular-lattice structures for various geometrical parameters and temporal pulses in order to explore the characteristics of broadband radiation and finally optimize it for a dedicated application with an effective bandwidth and desired power.

Out of a few C-PCF structures, we choose the ones with $R=1 \mu \mathrm{m}, 1.5 \mu \mathrm{m}, 2 \mu \mathrm{m}$ and $3 \mu \mathrm{m}$, respectively with $\underline{d / R}=0.9$. For all these cases, calculated dispersion curves are plotted in Fig. 11 and the nonlinearity parameters at the pump wavelengths were found to be $0.394 \mathrm{~W}^{-1} \mathrm{~m}^{-1}, 0.165 \mathrm{~W}^{-1} \mathrm{~m}^{-1}, 0.089 \mathrm{~W}^{-1} \mathrm{~m}^{-1}$ and

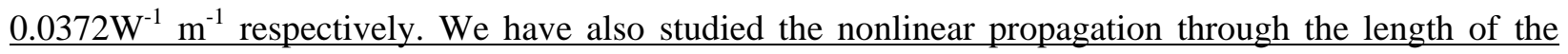
fiber. Fig. 12 shows the simulated nonlinear propagation towards the output as SC spectra in the visible light region in the PCF host $(R=1 \mu \mathrm{m}, d=0.9 \mu \mathrm{m})$. For the above figure, we used the input laser pulse of peak power $\left(\mathrm{P}_{0}\right) 1 \mathrm{~kW}, \mathrm{~T}_{\mathrm{FWHM}}$ as $200 \mathrm{fs}$ at the operating wavelength of $625 \mathrm{~nm}$ in a temporal window of 8 ps. This figure reveals the saturation of optical pulse towards generation of broad band in the visible region. The spectrum presented in the paper is obtained at the output end of the $20 \mathrm{~cm}$ fiber length. For the above case, it evolves to a sufficient spectrum after traversing a segment of 5-6 cm length. To minimize the loss due to propagation, the output can be extracted at such segment of the fiber. Also, to suppress the confinement loss, the studied fibers are considered with high air filling fraction.

The output power spectra due to propagation of 100 femto-second pulse for the studied C-PCF geometrical structure with above mentioned different $R(R=1 \mu \mathrm{m}, 1.5 \mu \mathrm{m}, 2 \mu \mathrm{m}$ and $3 \mu \mathrm{m})$ and $d / R=0.9$ are depicted in Fig. 13. The key results of our study of broadband SC generation for different structural parameters and ultra-short input pulses are presented In Table 1. So the above results with silica based CPCF yield higher power SCG for applications in the visible and near-IR wavelength region. Throughout our study we have used femto-second pulse laser and the pump wavelength was chosen to be in the anomalous region proximity of $Z D W$. This choice arose for achieving the solitonic fission as well as fourwave mixing suppression and higher-order dispersive waves amplification. Besides the above factor, at the ZDW the maximum energy-transfer occurs, which leads to efficient SCG. Principal contribution responsible for spectral broadening has been soliton fission and dispersive wave radiation. We can have a wider broadband of the spectrum with the same pump wavelength and pulsed width in anomalous dispersion region but with a cost of smoothness degradation of the output spectrum [25]. Our results of SCG for visible and near IR wavelengths are having a compressed wavelength band owing to the large slope of dispersion curve of C-PCF capable of generating the compact high power broadband radiation at the output. The above discussed new directions of possibilities for SCG in C-PCF would make useful contribution in the ongoing research of broadband generation in PCF and designing such sources.

\section{Conclusion and Discussions}

Detailed analyses of the guiding and modal properties of a PCF where air-holes are arranged in a circular pattern in the cladding with a silica background have been investigated. Different values of air-hole diameter and various circular radius have been considered, in order to show how the PCF properties are influenced by the geometric characteristics of the lattice. It is shown that circular-lattice PCFs with smaller values of radius $R(1 \mu \mathrm{m})$ and high $d / R(\geq 0.7)$ can be used as dispersion compensating fiber. For higher values of $R$, the dispersion parameter as well as the slope is mostly positive in the wavelength 
range considered. Dispersion parameter changes drastically if we increase the $R$ values form $1 \mu \mathrm{m}$ to 1.5 $\mu \mathrm{m}$ for a fixed value of $d / R$. A comparison has been performed between the triangular-lattice PCFs and CPCFs. A C-PCF has been found to be an excellent candidate for dispersion compensation and the structure comes out to be better than the triangular-PCF for both single wavelength and broadband dispersion compensation (around $1550 \mathrm{~nm}$ wavelength) which is unique property for inline dispersion compensation. Even with a matched dispersion with an adjustable T-PCF, the C-PCF comes out favorable with better RDS and CR values for the communication wavelength. Propagation loss with circular-lattice PCF comes out to be higher than the equivalent triangular-lattice PCF. However, the loss can always be reduced with increasing number of air-hole rings around the center. Effective area which is a very important parameter for various applications has been studied with different perspective. For equal values of hole-to-hole spacing and air-hole diameter the effective area of C-PCF was always found to be higher than the T-PCF. However, if we match the dispersion of the C-PCF at a particular wavelength with a different T-PCF with adjustable parameter, the corresponding effective area does not always remain higher for the C-PCF. In the later case the effective area for smaller wavelength is lower for C-PCF than the TPCF; however for higher wavelength the value is again higher for C-PCF. Again if we match the $\mathrm{A}_{\text {eff }}$ for the two fibers with adjustable parameter for a certain wavelength, the dispersion compensation of CPCF is always better than the corresponding T-PCF. With the above advantage along with its red-shifted ZDW and flatter slope in the anomalous dispersion region and lower value of GVD in the normal dispersion region, the new geometrical structure is preferable towards high power applications like supercontinuum generation, four wave mixing and parametric amplification and soliton pulse propagation. We also present a detailed analysis of SCG with C-PCF showing that such PCF geometry with fused silica compositions can be applied as a SC source in visible to mid-IR region with femtosecond laser pulse. We provide a series study of these C-PCF structures for different geometrical parameters and temporal pulses in order to explore the characteristics of broadband radiation that can be optimized towards a dedicated application with an effective bandwidth and power.

\section{Acknowledgment}

The authors would like to thank Dr. Boris Kuhlmey, University of Sydney, Australia for providing valuable suggestions in understanding the software for designing and studying the properties of different structures. Thanks are also due to Dr. Alessandro Tonello of Xlim Institute, UMR CNRS Limoges cedex, France for providing valuable suggestion in developing and understanding the NLSE codes. The authors acknowledge sincerely the Defence Research and Development Organization, Govt. of India and CRF of IIT Kharagpur for the financial support to carry out this research.

\section{Conflict of interest:}

The authors would like to declare that there is no direct financial relation with any commercial identity mentioned in their work that might lead to a conflict of interests.

\section{References:}

[1] J. C. Knight, "Photonic crystal fibres," Nature, 424, 847-851 (2003).

[2] P. St. J. Russel, "Photonic-Crystal Fibers," J of Lightwave Technol. 24, 4729-4749 (2006).

[3] B. T. Kuhlmey, G. Renversez and D. Maystre, "Chromatic dispersion and losses of microstructured optical fibers," Appl. Opt. OT 42, 634-639 (2003).

[4] A. H. Bouk, A. Cucinotta, F. Poli, S. Selleri "Dispersion properties of square-lattice photonic crystal fibers," Opt Express 12, 941-946 (2004) 
[5] W. H. Reeves, J. C. Knight, P. St. J. Russell, and P. J. Roberts, "Demonstration of ultra-flattened dispersion in photonic crystal fibers," Opt. Express 10, 609-613 (2002).

[6] K. Saitoh and M. Koshiba, "Chromatic dispersion control in photonic crystal fibers: application to ultraflattened dispersion," Opt. Express 11, 843-852 (2003)

[7] P. S. Maji and P. Roy Chaudhuri, "A New Design of Ultra-Flattened Near-zero Dispersion PCF Using Selectively Liquid Infiltration," Journal of Photonics and Optoelectronics, 2, 24-31(2013).

[8] P. S. Maji and P. Roy Chaudhuri, "Tunable fiber optic parametric amplifier based on near zero ultra flat dispersion PCF for communication wavelength," IEEE Photonics Journal, 7 (3), 1400311 (2015).

[9] P. S. Maji and P. Roy Chaudhuri, "Gain and bandwidth investigation in a near zero ultra-flat dispersion PCF for optical parametric amplification around the communication wavelength," Applied Optics. (OSA) 54. 11, 3263-3272 (2015).

[10] P. S. Maji and P. Roy Chaudhuri, "Supercontinuum generation in ultra-flat near zero dispersion PCF with selective liquid infiltration," Optik, International Journal of Light and Electro Optics (IJLEO), 125, 20, 59865992 (2014).

[11] P. S. Maji and P. Roy Chaudhuri, "Near Zero Ultra-flat Dispersion PCF: Properties and Generation of Broadband Supercontinuum," Journal of Photonics and Optoelectronics (P\&O), 3 51-58, (2014).

[12] P. S. Maji and P. Roy Chaudhuri, "Geometrical parameters dependence towards ultra-flat dispersion squarelattice PCF with selective liquid infiltration," American Journal of Optics and Photonics (AJOP), 1, 5, 28-32, 2013, doi: 10.11648/j.ajop.20130105.11

[13] X. Zhao, G. Zhou, S. Li, Z. Liu, D. Wei, Z. Hou, and L. Hou, "Photonic crystal fiber for dispersion compensation," Appl. Opt.47, 5190-5196 (2008).

[14] S. Yang, Y. Zhang, X. Peng, Y. Lu, A. Xie, J. Li, W. Chen, Z. Jiang, J. Peng, and H. Li, "Theoretical study and experimental fabrication of high negative dispersion photonic crystal fiber with large area mode field," Opt. Express 14, 3015-3023 (2006).

[15] F. Gérôme, J.-L. Auguste, and J.-M. Blondy, "Design of dispersion-compensating fibers based on a dualconcentric-core photonic crystal fiber," Opt. Lett. 29, 2725-2727 (2004).

[16] P. S. Maji and P. Roy Chaudhuri, "ASE suppression in Er3+ doped dual-core triangular lattice Photonic Crystal Fibers (PCFs) for narrowband and broadband dispersion compensation for communication wavelength ," Optik, International Journal of Light and Electro Optics (IJLEO), 127, 1, 292-298 (2016).

[17] P. S. Maji and P. Roy Chaudhuri, "Designing broad band dispersion compensation with square lattice PCF and applications to ASE suppression with ultra negative dispersion," Optik, International Journal of Light and Electro Optics (IJLEO), 127, 5, 2603-2607 (2016).

[18] P. S. Maji and P. Roy Chaudhuri, "Design of ultra large negative dispersion PCF with selectively tunable liquid infiltration for dispersion compensation," Optics Communications, 325, 134-143 (2014).

[19] P. S. Maji and P. Roy Chaudhuri, "Designing an Ultra-Negative Dispersion Photonic Crystal Fiber with SquareLattice Geometry," ISRN Optics, vol. 2014, 7 pages, Article ID 545961, 2014. doi:10.1155/2014/545961

[20] Wei-De Ho, Tsan-Wen Lu, Yi-Hua Hsiao, and Po-Tsung Lee, "Thermal Properties of 12-Fold Quasi-Photonic Crystal Microcavity Laser With Size-Controlled Nano-Post for Electrical Driving" J of Lightwave Technol. 27, 5302-5307 (2009).

[21] N. Horiuchi, Y. Segawa, T. Nozokido and K. Mizuno H. Miyazaki, "High-transmission waveguide with a small radius of curvature at a bend fabricated by use of a circular photonic crystal," Opt. Lett. 30, 973-975 (2005).

[22] Po-Tsung Lee, Tsan-Wen Lu, Jyun-Hao Fan, and Feng-Mao Tsai, "High quality factor microcavity lasers realized by circular photonic crystal with isotropic photonic band gap effect," Appl. Phy. Lett. 90, 151125151125-3 (2007).

[23] Wei Zhong and Xiangdong Zhang, "Localized modes in defect-free two-dimensional circular photonic crystals," Phy. Rev. A 81, 013805-013805-6 (2010).

[24] Huizhen Xu, JianWu, Kun Xu, Yitang Dai, Cong Xu and Jintong Lin "Ultra-flattened chromatic dispersion control for circular photonic crystal fibers," J. Opt. 13, 055405-055405-7 (2011).

[25] J. Xu, J. Song, C. Li, and K. Ueda, "Cylindrically symmetrical hollow fiber", Opt. Commun. 182, 343-348 (2000).

[26] V. Rastogi and K. S. Chiang, "Holey optical fiber with circularly distributed holes analyzed by the radial effective-index method", Opt. Lett, 28, 2449-2451 (2003).

[27] P. S. Maji and P. Roy Chaudhuri, "Circular Photonic Crystal Fibers: Numerical Analysis of Chromatic Dispersion and Losses," ISRN Optics, Vol. 2013, 9 pages, Article ID, 986924, 2013. doi:10.1155/2013/986924

[28] A. Argyros, I. M. Bassett, M. A. van Eijkelenborg, M. C.J. Large, J. Zagari N. A.P. Nicorovici, R. C. McPhedran and C. M. de Sterke, "Ring structures in microstructured polymer optical fibres," Opt. Express. 9 
813-820 (2001).

[29] CUDOS MOF utilities available online: http://www.physics.usyd.edu.au/cudos/mofsoftware/

[30] T. P. White, B. T. Kuhlmey, R. C. PcPhedran, D. Maystre, G. Renversez, C. M de Sterke and L. C. Botten, "Multipole method for microstructured optical fibers. I. Formulation" J. Opt. Soc. Am. B. 19, 2322-2330 (2002).

[31] B. T. Kuhlmey, T. P. White, R. C. PcPhedran, D. Maystre, G. Renversez, C. M de Sterke and L. C. Botten, "Multipole method for microstructured optical fibers. II. Implementataion and results." J. Opt. Soc. Am. B. 19, 2331-2340 (2002).

[32] T. A. Birks, D. Mogilevtsev, J. C. Knight, and P. St. J. Russell, “Dispersion compensation using single-material fibers,' IEEE Photonics Technol. Lett. 11, 674-676 (1999).

[33] 'CCorning® SMF-28 CPC6 Single-Mode Optical Fibre,' Product Information (Corning, Ithaca, N.Y., 1998).

[34] J. M. Dudley, G. Genty, S. Coen, "Supercontinuum generation in photonic crystal fiber," Rev. Mod. Phys. 78, 1135-1184 (2006).

[35] G. P. Agrawal, Nonlinear Fiber Optics, 4th ed., Optics and Photonics Series (Academic, San Diego, Calif., 2007).

[36] V. L. Kalashnikov, E. Sorokin, I. T. Sorokina, "Raman effects in the infrared supercontinuum generation in softglass PCFs," Appl. Phys. B. 87, 37-44 (2007).

[37] P. S. Maji and P Roy Chaudhuri, "A new design of all normal dispersion based on multi-material PCF in IR region for broadband supercontinuum generation," Applied Optics. (OSA) 54. 13, 4042-4048 (2015). 


\section{FIGURES}

Partha et.al Fig. 1/13

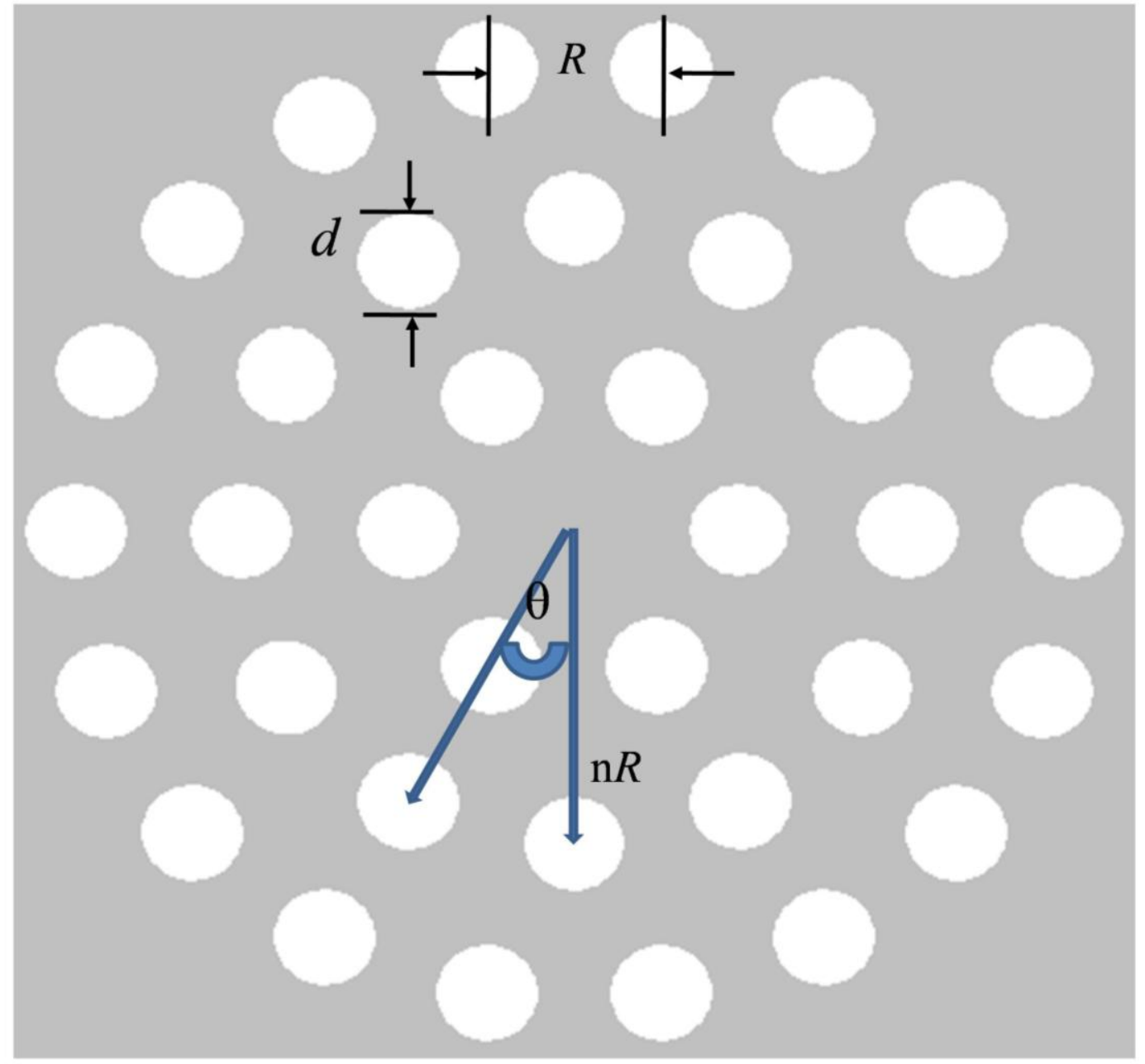


Partha et.al Fig. 2/13

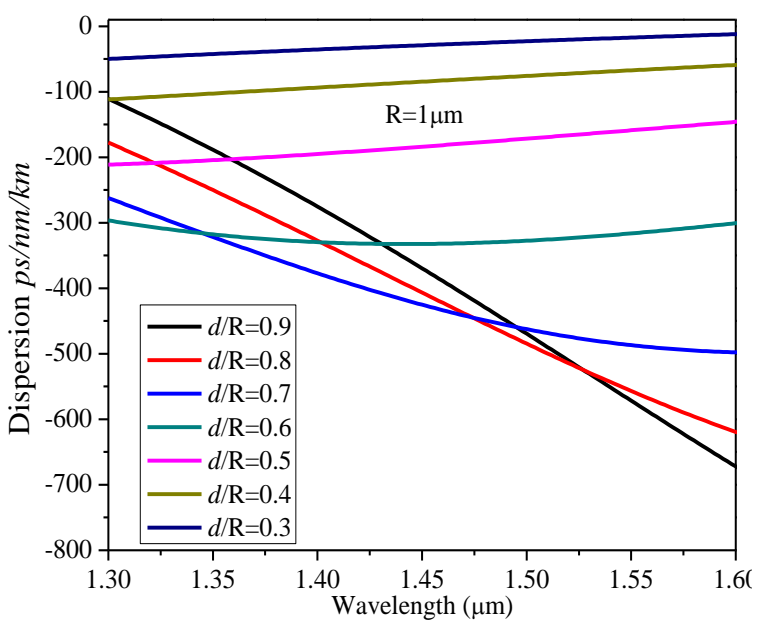

(a)

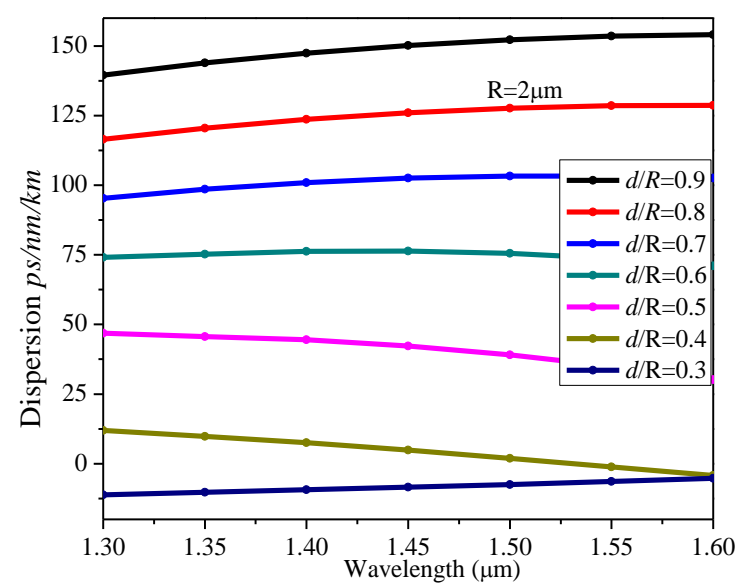

(b)

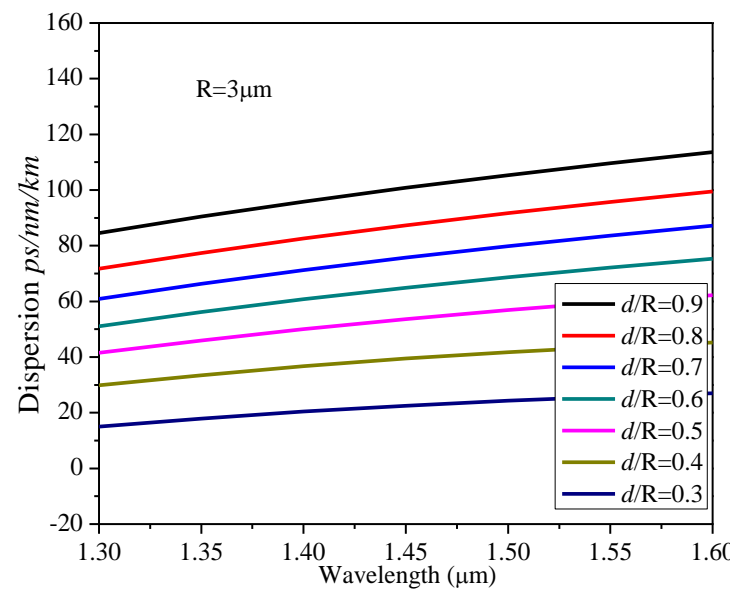

(c) 


\section{Partha et.al Fig. 3(a)/13}

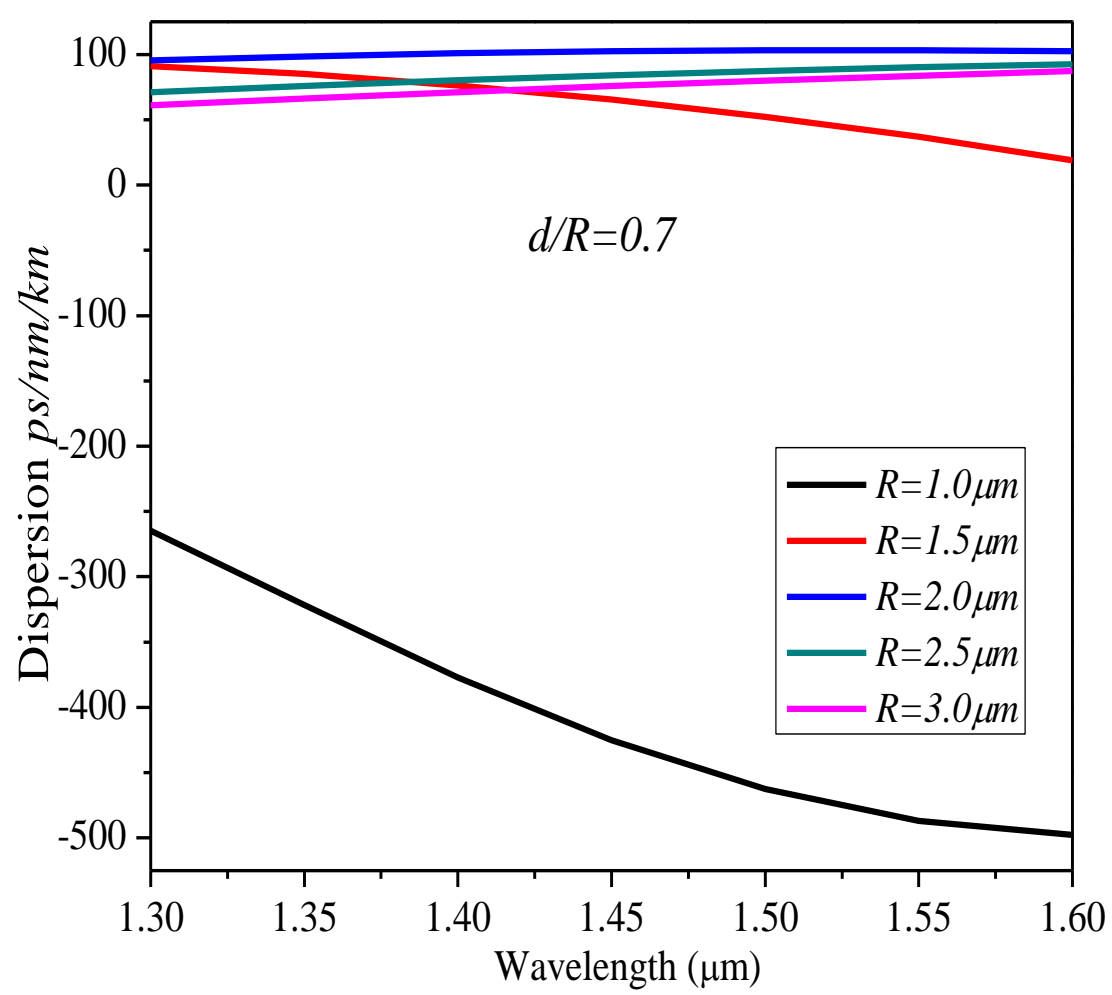

Partha et.al Fig. 3(b)/13

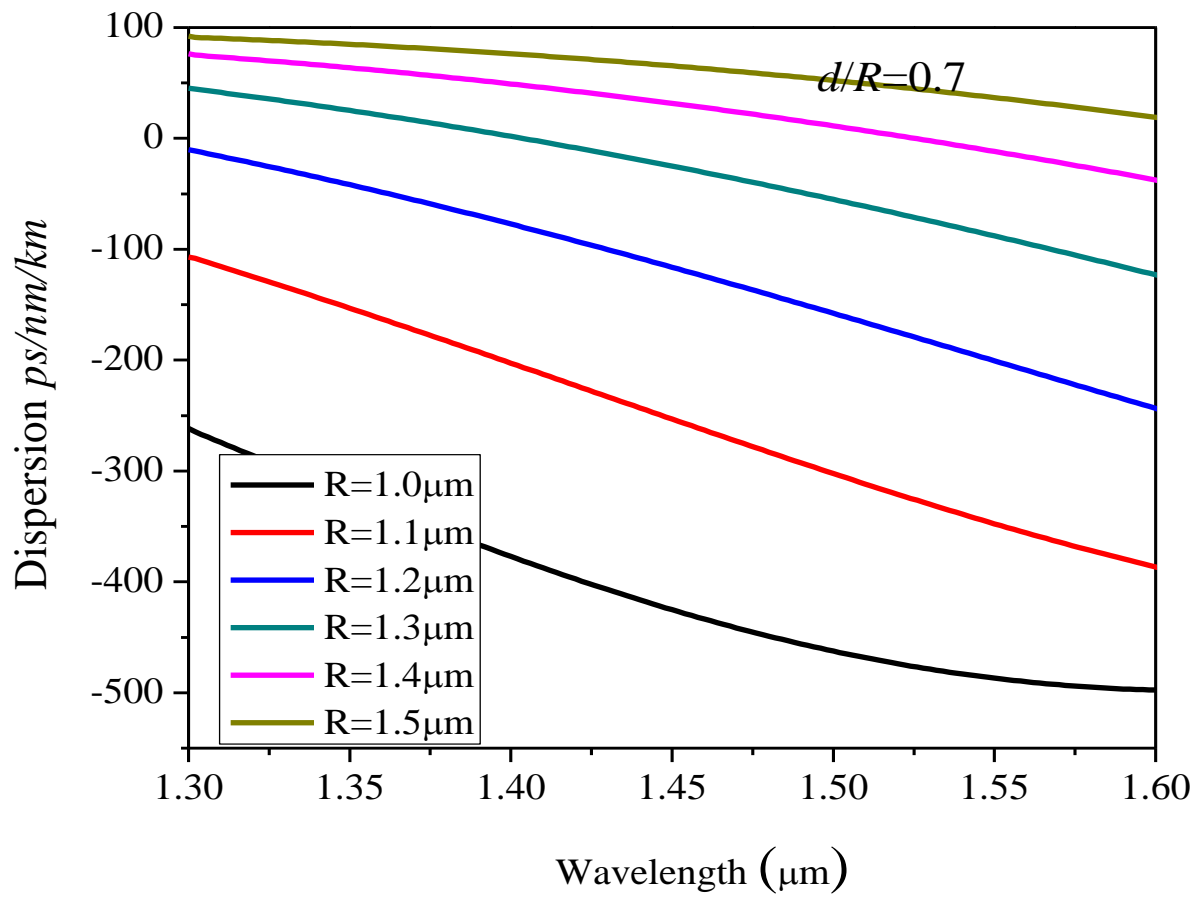




\section{Partha et.al Fig. 4(a)/13}

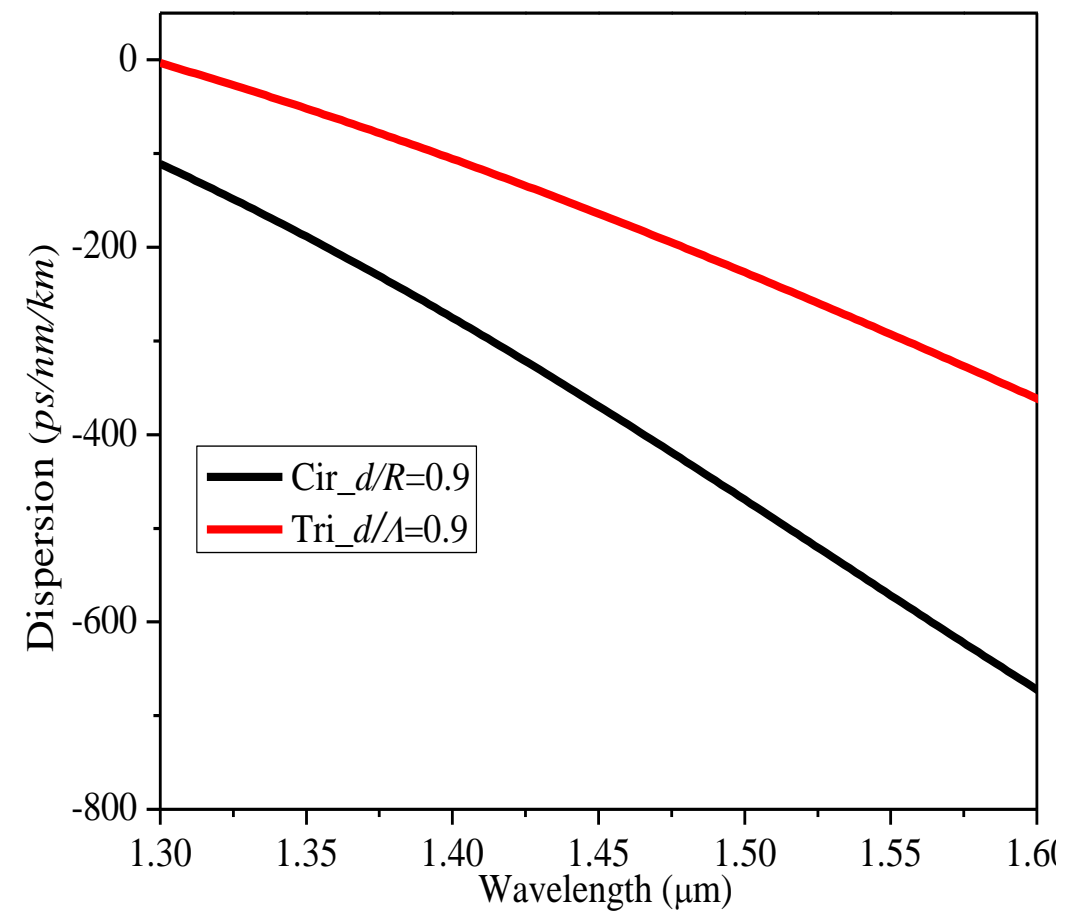

Partha et.al Fig. 4(b)/13

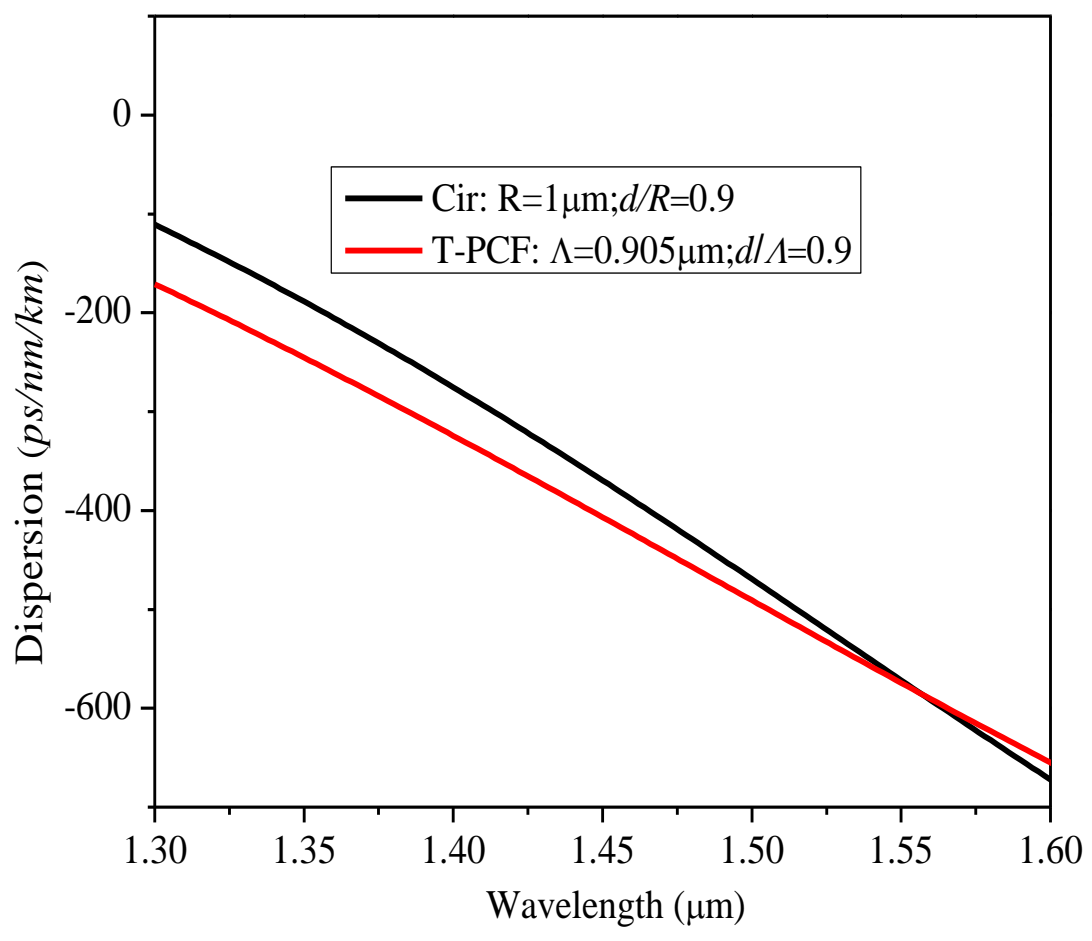


Partha et.al Fig. 5(a)/13

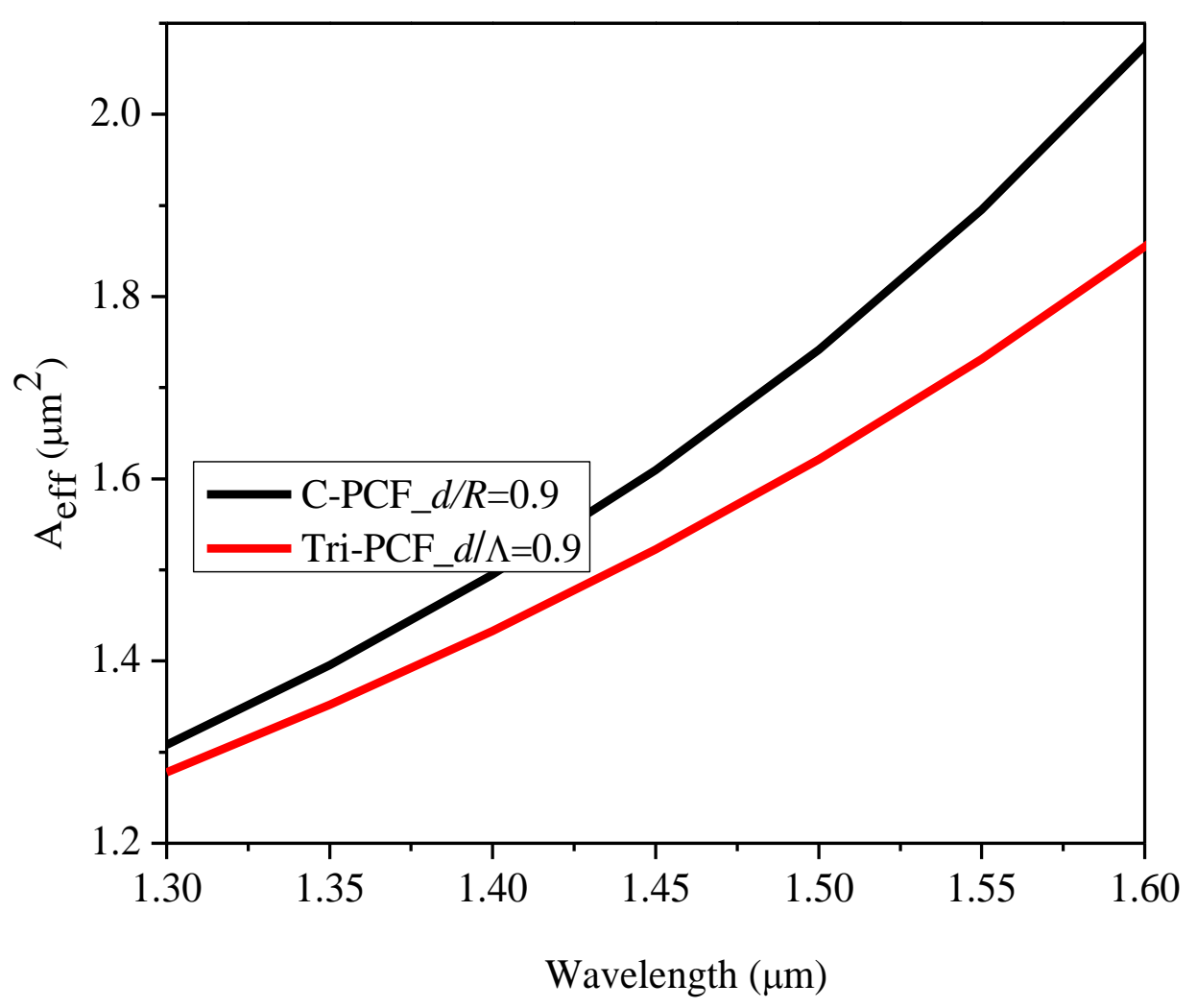

Partha et.al Fig. 5(b)/13

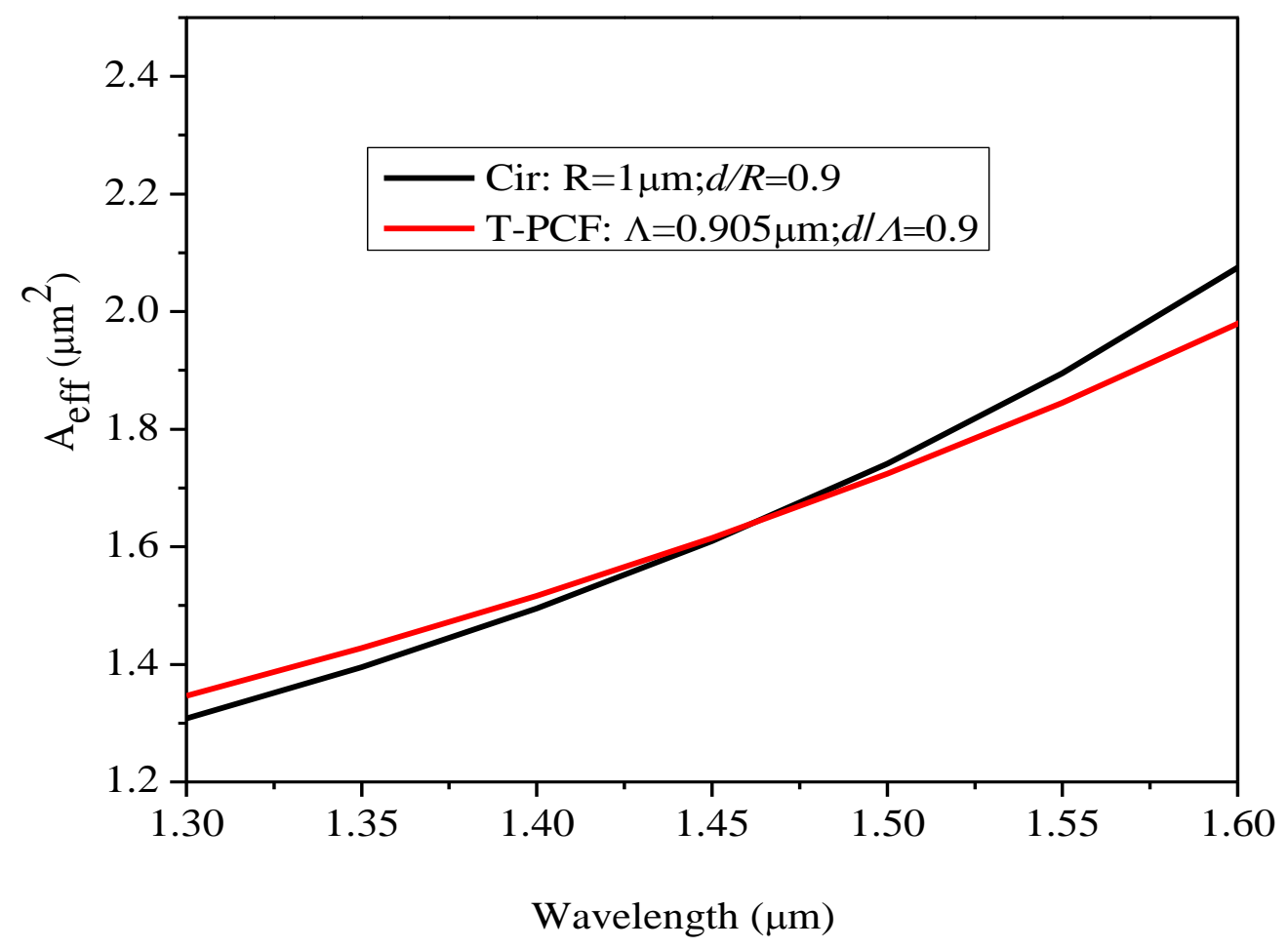




\section{Partha et.al Fig. 6(a)/13}

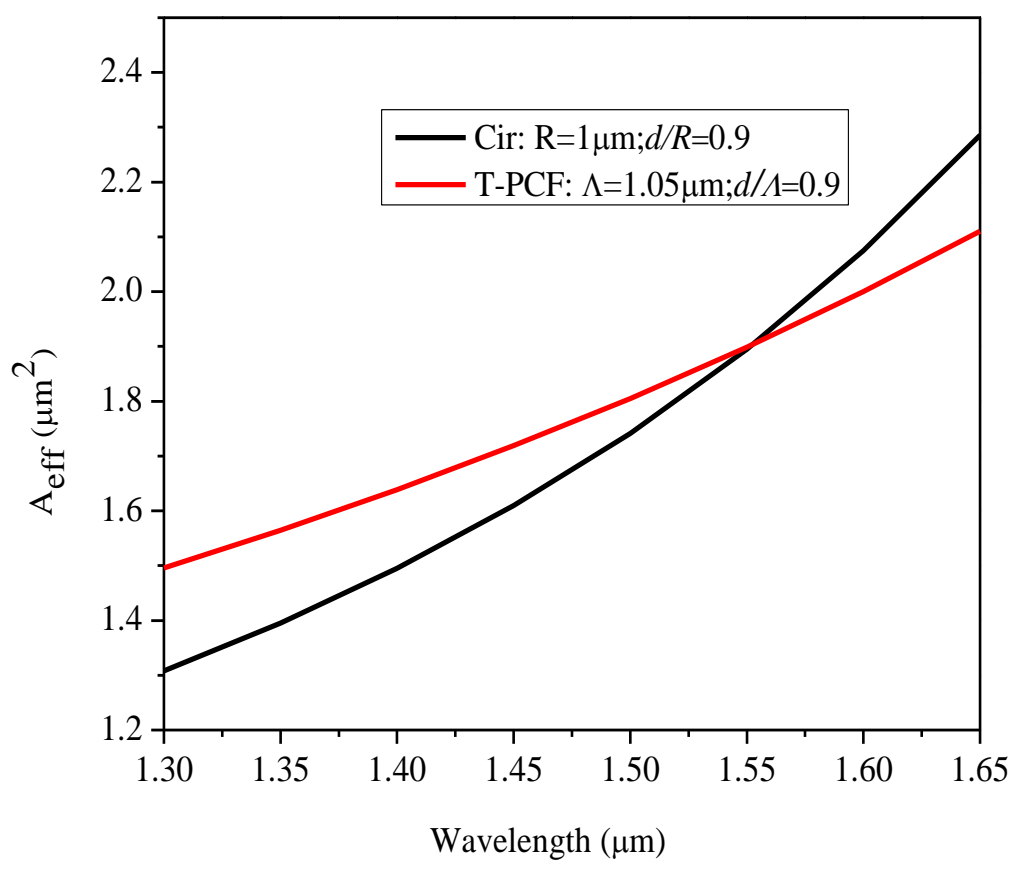

\section{Partha et.al Fig. 6(b)/13}

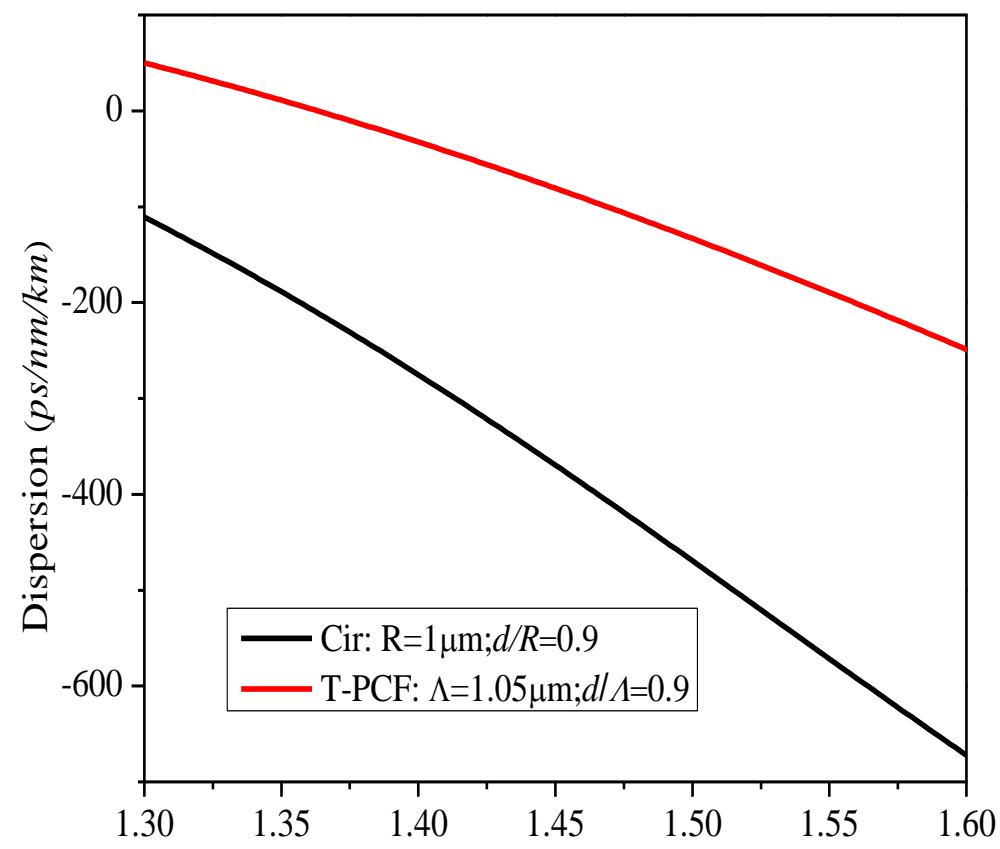


Partha et.al Fig. 7(a)/13

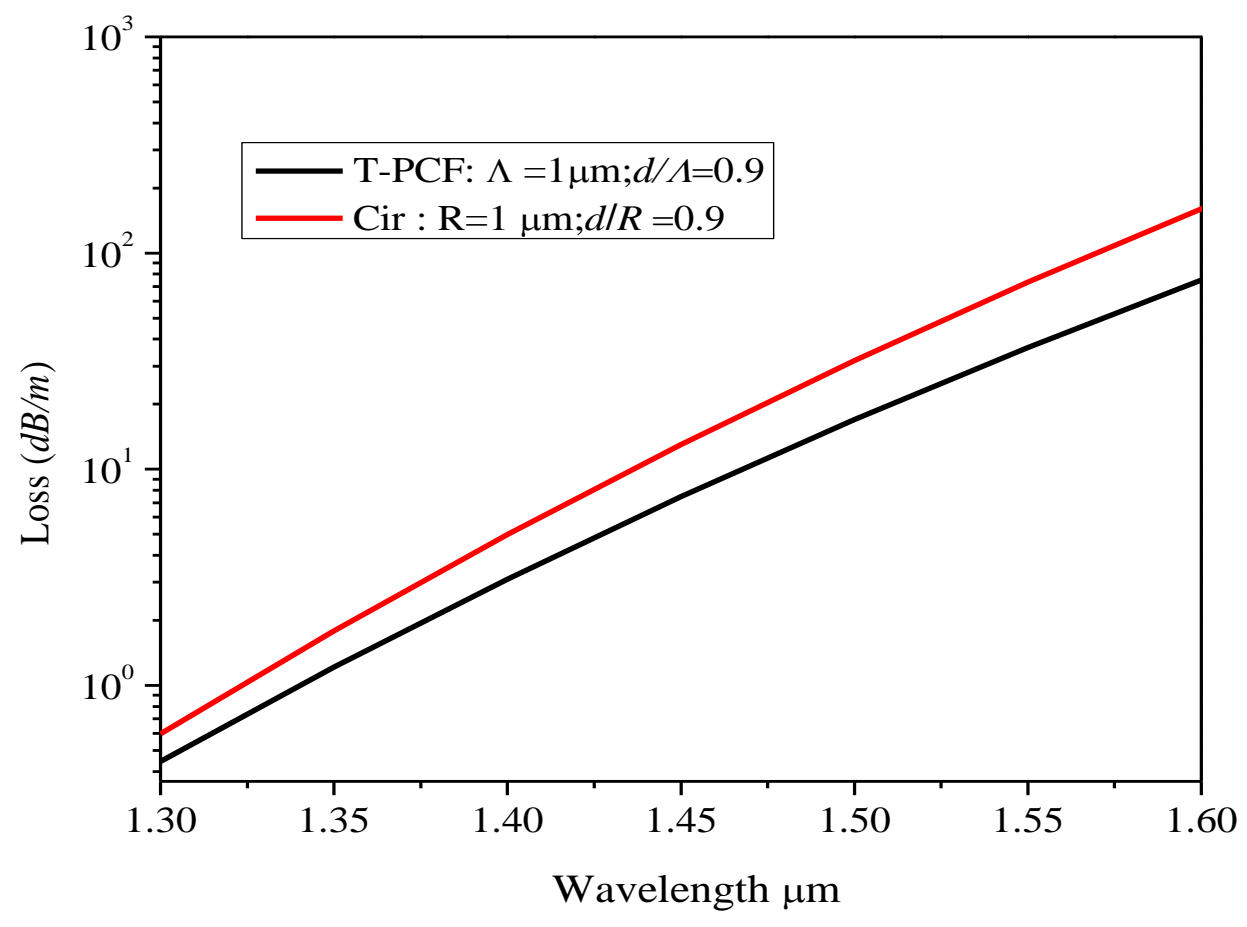

Partha et.al Fig. 7(b)/13

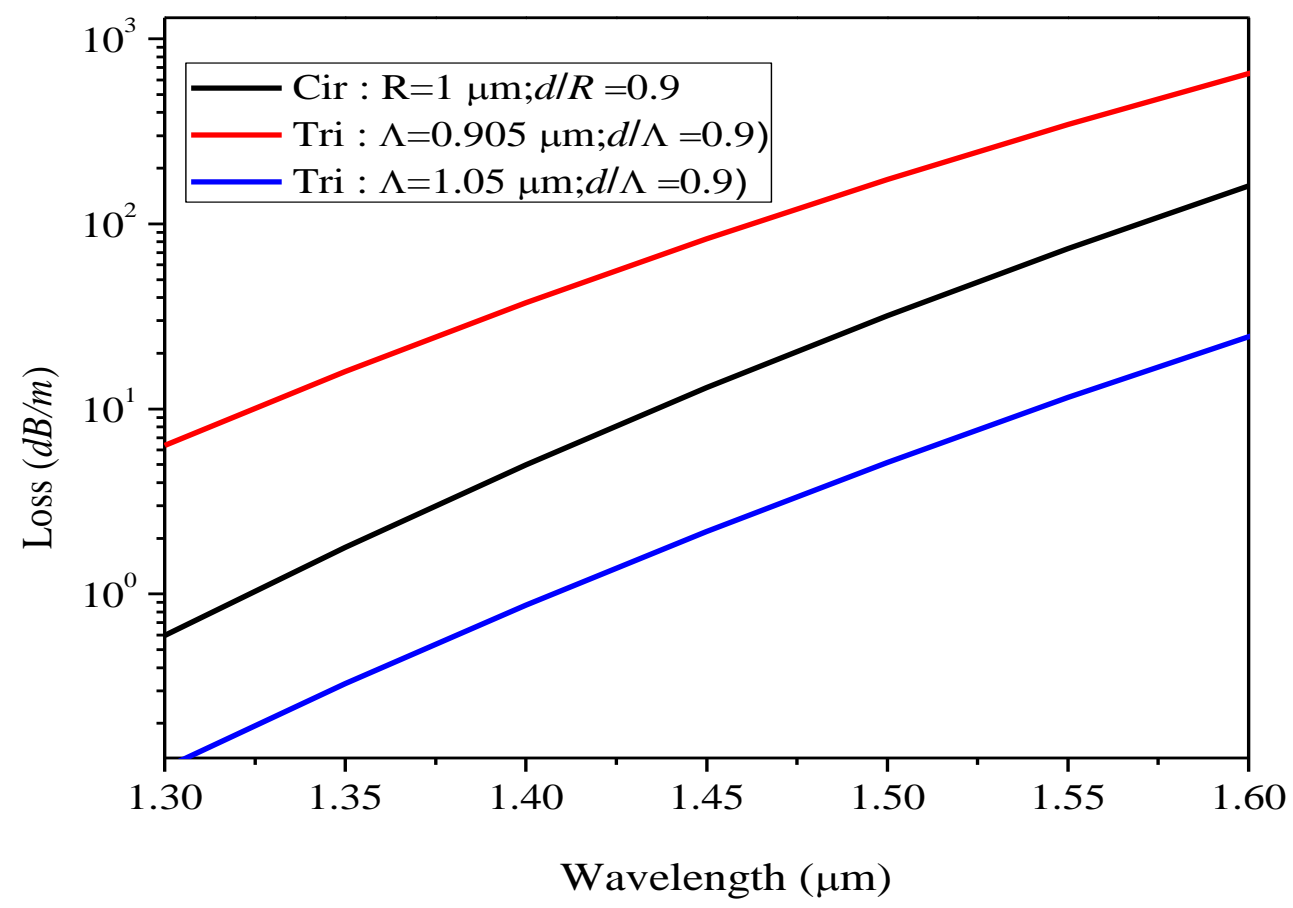




\section{Partha et.al Fig. 8/13}

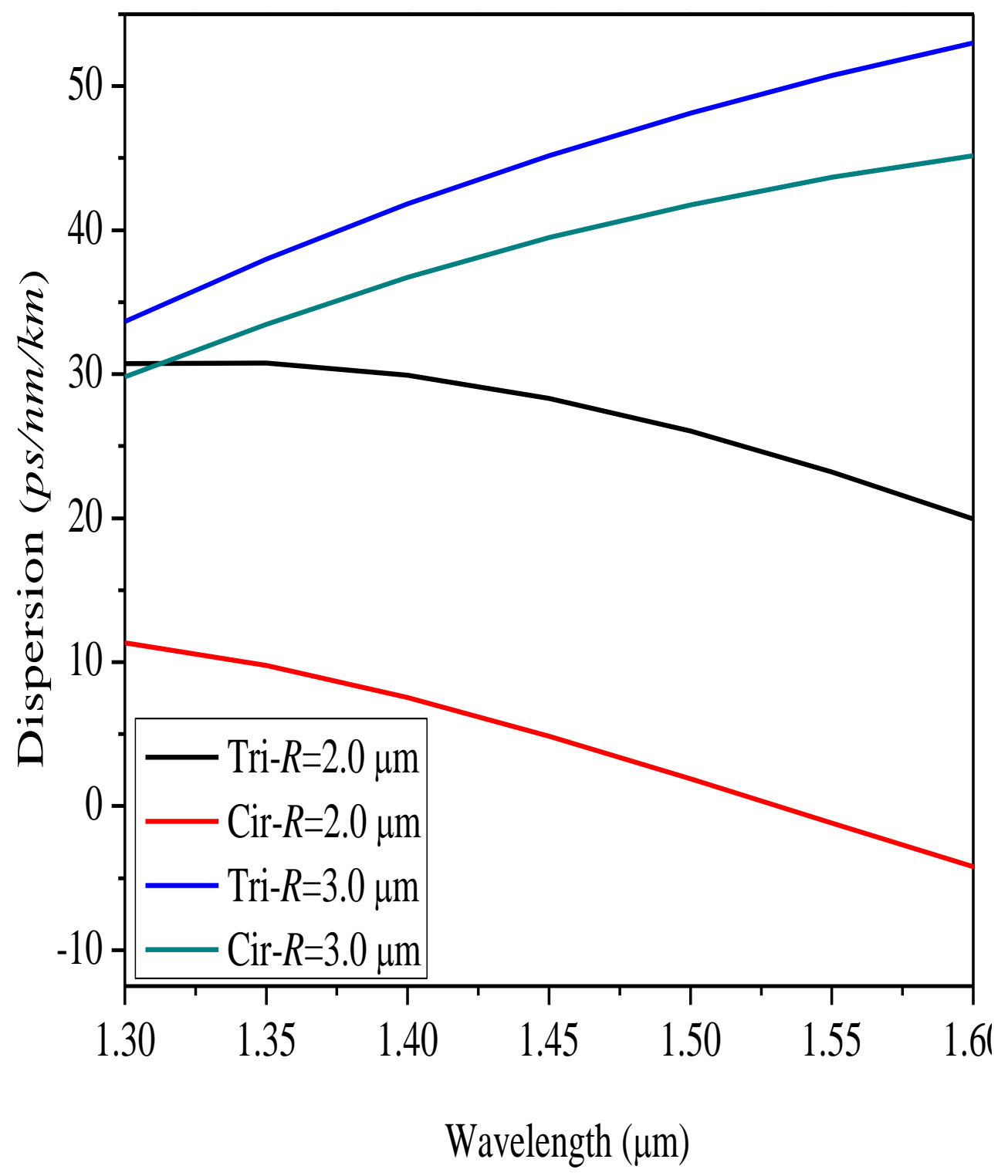


Partha et.al Fig. 9/13

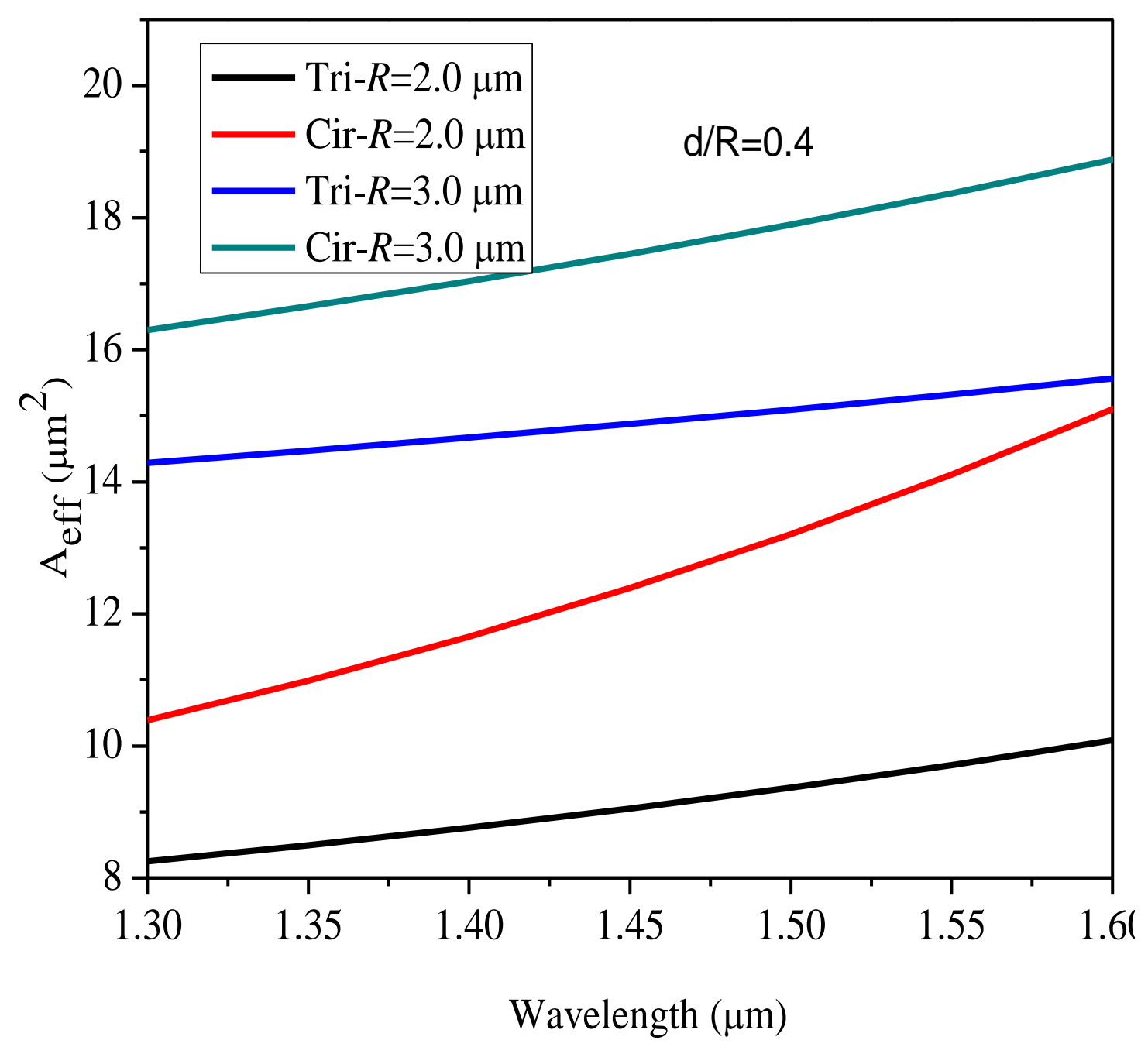


Partha et.al Fig. 10/13

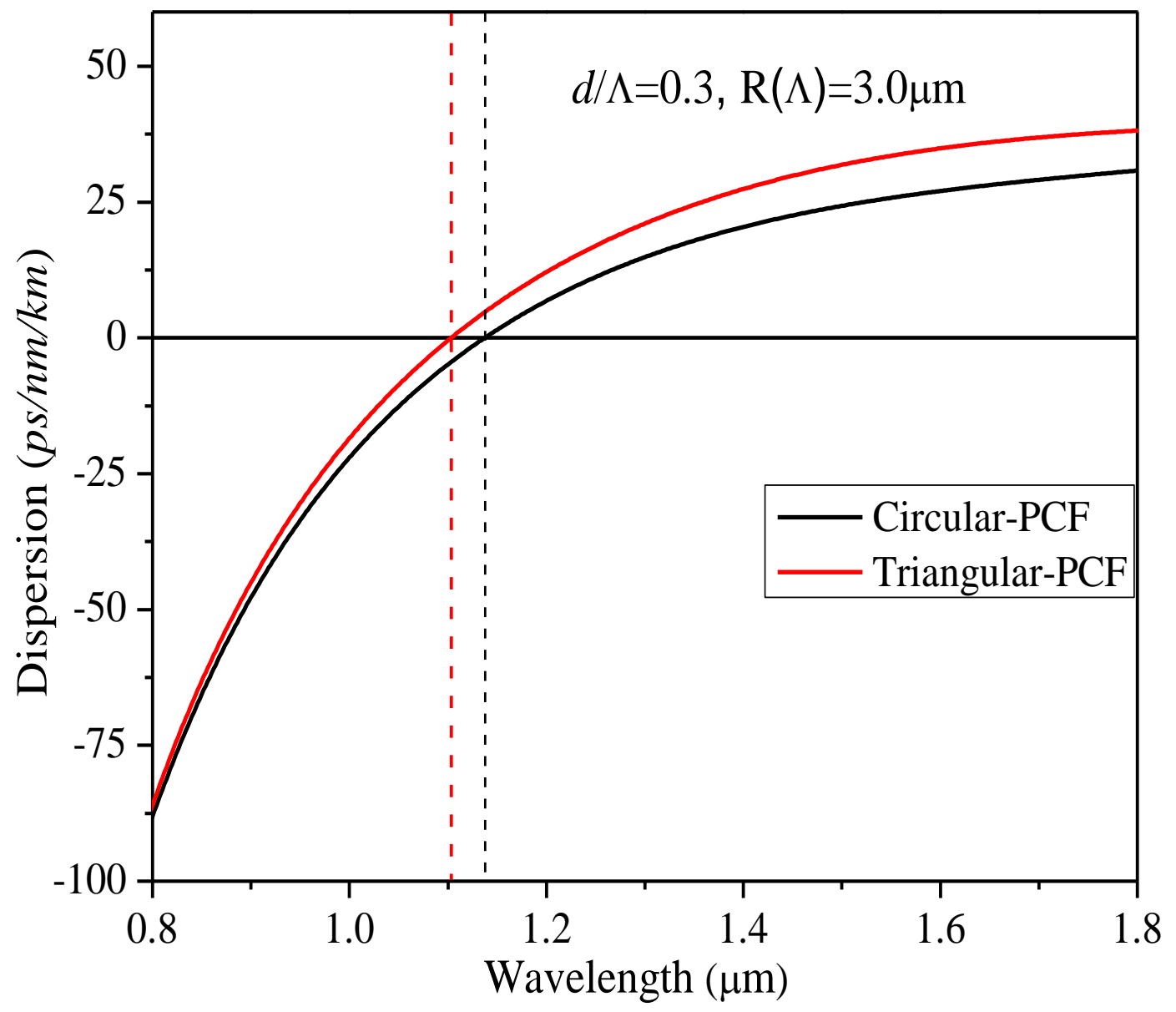


Partha et.al Fig. 11/13

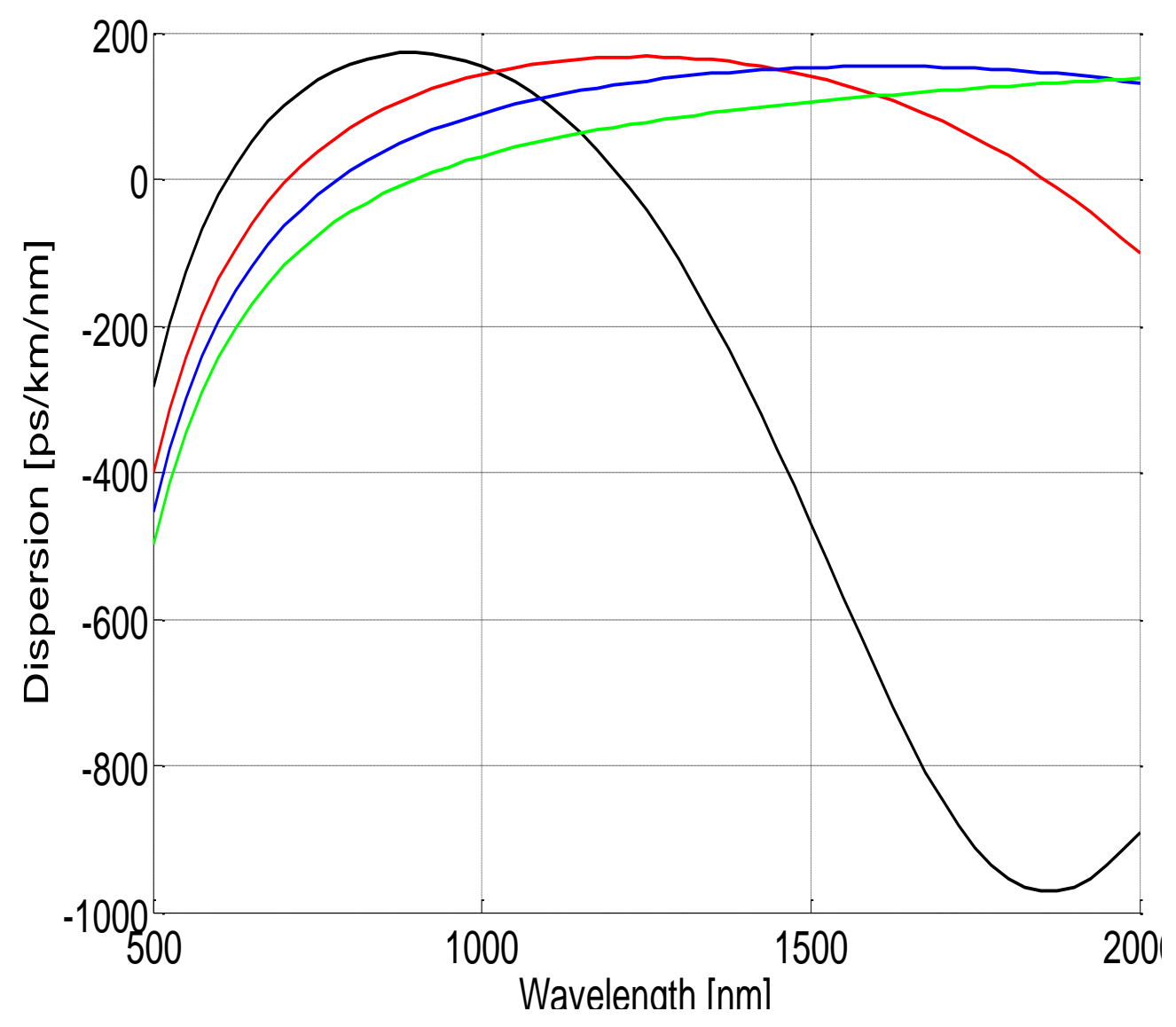


Partha et.al Fig. 12/13

Propagation, spectral domain

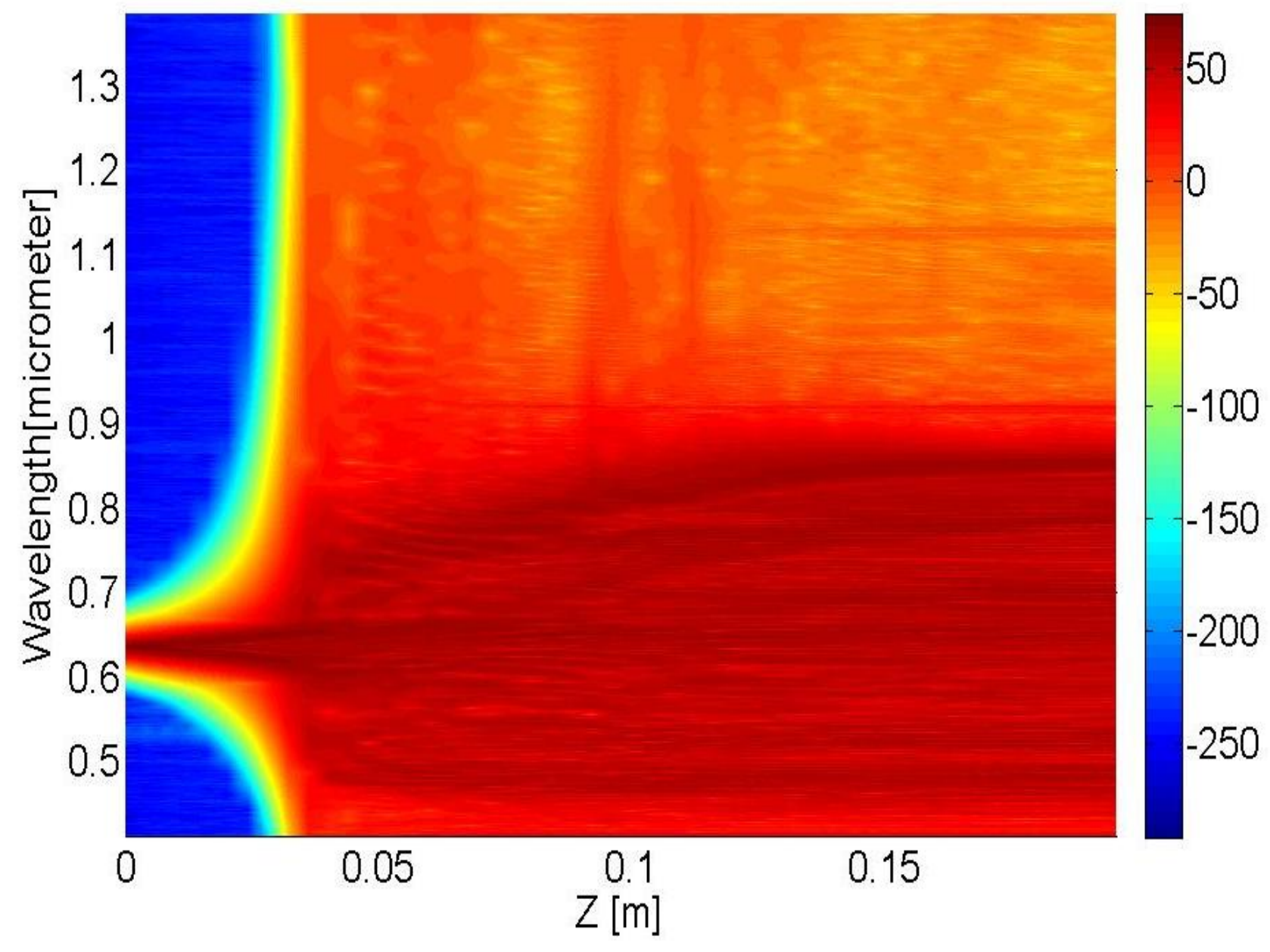


Partha et.al Fig. 13/13

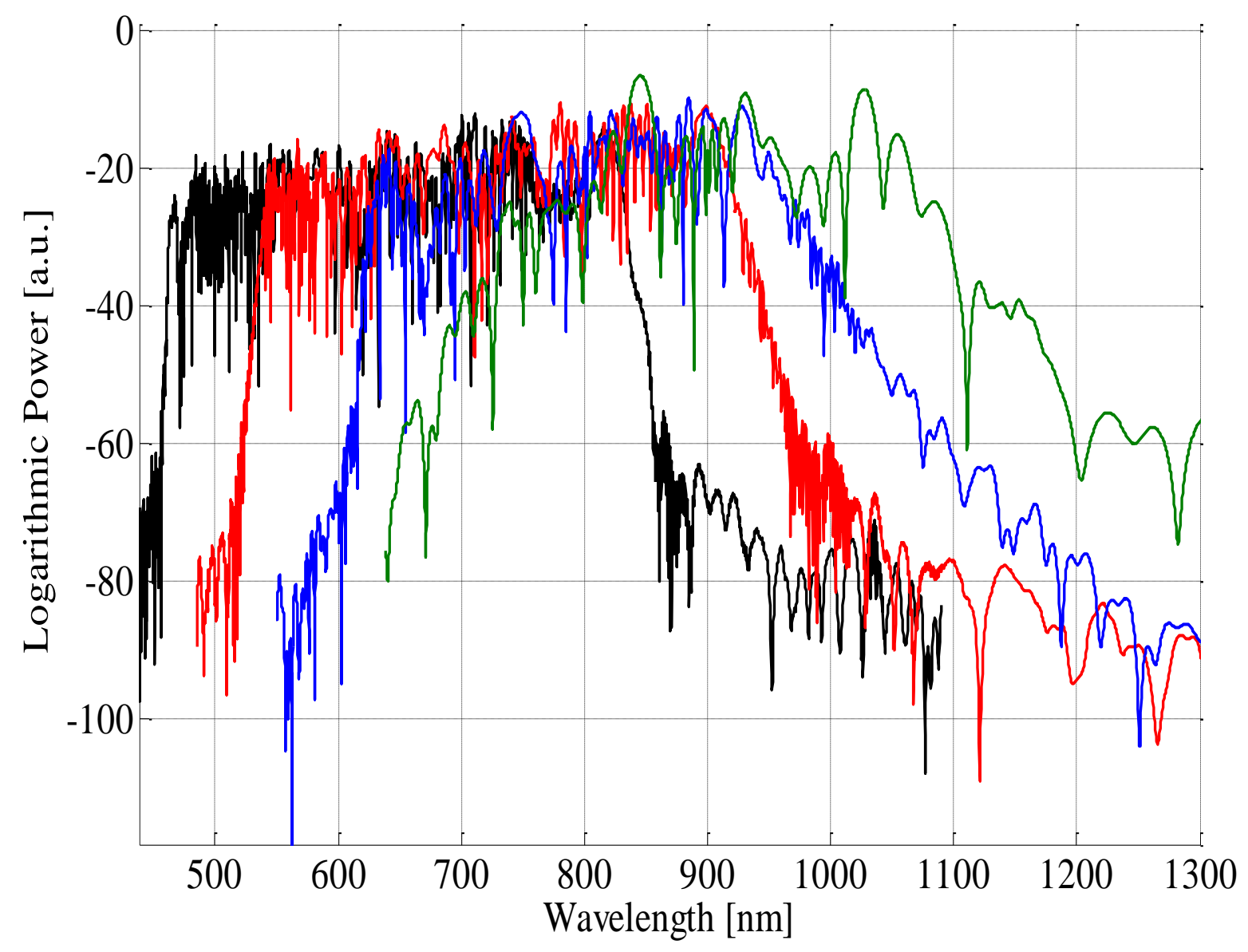




\section{FIGURE (PHOTO) DESCRIPTION}

Fig. 1: The schematic diagram of the studied fiber. The spacing between the air-holes in a particular circle is $R$ and the distance from the center to $\mathrm{n}^{\text {th }}$ circle is $\mathrm{n} R$ with silica background.

Fig. 2: Dispersion curves of the C- PCFs with (a) $R=1 \mu \mathrm{m}$, (b) $R=2 \mu \mathrm{m}$ and (c) $R=3 \mu \mathrm{m}$ for different $d / R$ values.

Fig. 3: (a) Dispersion curves of the circular-lattice PCF with $d / R=0.7$ for different values of $R$. (b) A better mapping of the dispersion of Fig. 3(a) with $R$ changes by step of $0.1 \mu \mathrm{m}$ for $R$ from $1.0 \mu \mathrm{m}$ and $1.5 \mu \mathrm{m}$.

Fig. 4: (a) Comparison of the dispersion parameter for the C-PCF with triangular-lattice PCF with $d / \Lambda=$ $0.9(d / R=0.9$ for C-PCF $)$ and $\Lambda(R$ for C-PCF $)=1 \mu \mathrm{m}$. (b) A new T-PCF with $\Lambda=0.905 \mu \mathrm{m}$ and $d / \Lambda=0.9$ demonstrates similar dispersion value with the C-PCF (with $\mathrm{R}=1 \mu \mathrm{m}$ and $d / \Lambda=0.9$ ) around $1550 \mathrm{~nm}$ of wavelength.

Fig. 5: (a) Comparison of the Effective area for the C-PCF with triangular-lattice PCF with $d / \Lambda=0.9$ $(d / R=0.9$ for $\mathrm{C}-\mathrm{PCF})$ and $\Lambda(R$ for $\mathrm{C}-\mathrm{PCF})=1 \mu \mathrm{m}$. (b)Effective area variation corresponding to Fig. 4(b) for $\Lambda=0.905 \mu \mathrm{m}$ and $d / \Lambda=0.9$.

Fig. 6: (a) A new T-PCF with $\Lambda=1.05 \mu \mathrm{m}$ and $d / \Lambda=0.9$ demonstrates similar dispersion value with the C-PCF (with $\mathrm{R}=1 \mu \mathrm{m}$ and $d / \Lambda=0.9$ ) around $1550 \mathrm{~nm}$ of wavelength. (b) Dispersion nature corresponding to Fig. 6(a) for T-PCF with $\Lambda=1.05 \mu \mathrm{m}$ and $d / \Lambda=0.9$.

Fig. 7: Comparison of the propagation loss for (a) C-PCFs with triangular-lattice PCF with $\mathrm{R}(\Lambda)=1 \mu \mathrm{m}$ and $d / R$ (or $d / \Lambda)=0.9$, (b) C-PCFs having $R=1 \mu \mathrm{m}$ and $d / R=0.9$, with T-PCF having $\Lambda=0.905$ $\mu \mathrm{m}$ and $d / \Lambda=0.9$ (that demonstrate same dispersion at $1550 \mathrm{~nm}$ as per Fig. $4(\mathrm{~b})$ ) and $\Lambda=1.05 \mu \mathrm{m}$ and $d / \Lambda=0.9$ (that demonstrate equal $\mathrm{A}_{\text {eff }}$ at $1550 \mathrm{~nm}$ as per Fig. 6(a)).

Fig. 8: Comparison of the dispersion properties for the C-PCFs with triangular-lattice PCF with $d / \Lambda$ (or $d / R)=0.4$, for $\Lambda(R)=2 \mu \mathrm{m}$ and $\Lambda(R)=3 \mu \mathrm{m}$.

Fig. 9: Comparison of the effective area for the C-PCFs with triangular-lattice PCF with $d / \Lambda$ (or $d / R)=$ 0.4 , for $\Lambda(R)=2 \mu \mathrm{m}$ and $\Lambda(R)=3 \mu \mathrm{m}$.

Fig. 10: Extension of Fig. 6(a) for wider range of wavelengths for a comparison of the dispersion properties of C-PCFs with triangular-lattice PCF with $d / \Lambda$ (or $d / R)=0.3$, for $\Lambda(R)=3 \mu \mathrm{m}$.

Fig. 11: Calculated dispersions in Fused silica CPCFs of geometrical parameters $\mathrm{R}=1.0 \mu \mathrm{m}$, (black); $R=$ $1.5 \mu \mathrm{m}$, (red) $R=2.0 \mu \mathrm{m}$, (blue) and $R=3.0 \mu \mathrm{m}$ (green) with $d / R=0.9$

Fig. 12: Evolution of optical pulse through length of the fiber for Fused Silica C-PCFs of geometrical parameters $R=1.0 \mu \mathrm{m}$ with $d / R=0.9$ and the corresponding color-bar. 
Fig.13: Output spectrum of the fiber corresponding to Fig. 11 with $100 \mathrm{fs}$ pulse. The colours are as per mentioned in Fig. 11. 


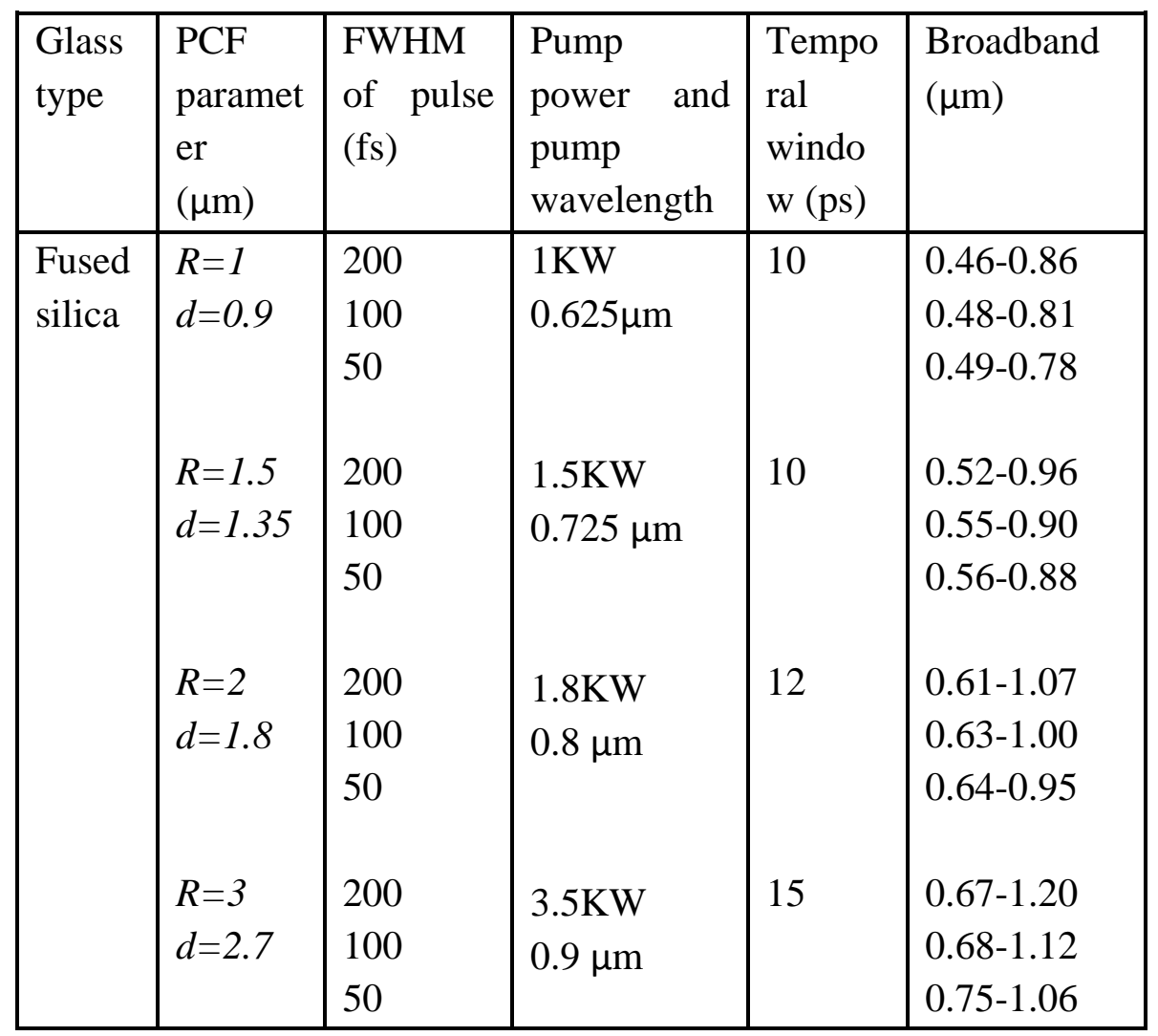




\section{TABLE DESCRIPTION}

TABLE 1: Summary of the SCG results with fused silica glasses with C-PCF geometry with 100 fs pulse. 